\title{
Fermionic partition functions for a periodic soliton cellular automaton
}

\author{
Atsuo Kuniba \\ Institute of Physics, University of Tokyo, Tokyo 153-8902, Japan \\ E-mail: atsuo@gokutan.c.u-tokyo.ac.jp \\ Taichiro Takagi \\ Department of Applied Physics, National Defense Academy, Kanagawa 239-8686, \\ Japan \\ E-mail: takagi@nda.ac.jp
}

\begin{abstract}
Fermionic formulas in combinatorial Bethe ansatz consist of sums of products of $q$-binomial coefficients. There exist refinements without a sum that are known to yield partition functions of box-ball systems with a prescribed soliton content. In this paper, such a refined fermionic formula is extended to the periodic box-ball system and a $q$-analogue of the Bethe root counting formula for XXZ chain at $\Delta=\infty$.
\end{abstract}

PACS numbers: 05.45.Yv, 02.30.Ik, 02.20.Uw 


\section{Introduction}

\subsection{Main result}

Combinatorial aspects of integrable systems have attracted considerable interest, constituting the field sometimes called "physical combinatorics" (see e.g. [1, 2]). In this paper we establish a new fermionic formula (Theorem 16):

$$
\sum_{p \in P_{L, \lambda}^{\circ}} q^{E_{\mathrm{path}}(p)}=q^{\psi(\lambda)} \frac{[L]}{\left[p_{1}\right]} \prod_{i=1}^{s}\left[\begin{array}{c}
p_{i}+m_{i}-1 \\
m_{i}
\end{array}\right],
$$

where $q$ is an indeterminate, $L$ is a positive integer and $\lambda$ is a Young diagram such that $|\lambda| \leq L / 2$. The numbers $m_{i}$ and $s$ are determined from $\lambda$ by Figure 1, from which $p_{i}$ (2) and $\psi(\lambda)$ (3) are also specified. The bracket symbols in the right hand side are defined in (8).

The left hand side of (11) is a partition function of the length $L$ periodic box-ball system (section 3.1) with a prescribed soliton content $\lambda$. A state $p$ of the box-ball system, we call it a path in this paper, is just an array of 1 and 2. The paths are subject to commuting time evolutions $T_{1}, T_{2}, \ldots$. See Example 11. Regarding 1's as background, one observes that there are solitons (consecutive 2's) with amplitudes 4,3,2,1 moving to the right under the periodic boundary condition. They get reshuffled locally and temporarily under the collisions but regain their original amplitudes after the events. In fact the list of properly defined amplitudes are known to be conserved and that defines the soliton content \pm . The $P_{L, \lambda}^{\circ}$ in (1) denotes the set of length $L$ paths having the soliton content $\lambda$. Finally $E_{\text {path }}(p)$, the energy of a path $p$, is given in Definition 4 .

\subsection{Background and related results}

As a guide to the formula (1), we recall some background and related results obtained earlier. We shall concentrate on the $s l_{2}$ case throughout the paper.

Generating functions of energy over paths, denoted by $X$, are known as the onedimensional configuration sums in Baxter's corner transfer matrix method [3]. On the other hand, the combinatorial version [4, 5] of the Bethe ansatz [6] provides the same quantity with another counting based on string hypothesis. It leads to the so called fermionic form $\mathcal{M}$. Then the physically suggested identity $X=\mathcal{M}$ can indeed be proved by the Kerov-Kirillov-Reshetikhin (KKR) bijection [4, 5] between paths and rigged configurations which are combinatorial objects labeling the monomials in $\mathcal{M}$. See for example the reviews by M. Okado and A. Schilling in [2] and references therein.

The fermionic form is actually a sum $\mathcal{M}=\sum_{\lambda} \mathcal{M}(\lambda)$, where each summand is a product of $q$-binomial coefficients reflecting the fermionic nature of Bethe roots. The sum gathers the contributions from all possible decompositions of the Bethe roots into strings. The $\lambda$ in the sum is a Young diagram called configuration labeling such string contents. A natural problem is then to refine the identity $X=\mathcal{M}$ to $X(\lambda)=\mathcal{M}(\lambda)$. By

$\ddagger$ It is $\lambda=(4321)$ in Example 11 . 
the construction, the paths responsible for $X(\lambda)$ are the restriction of the image of the KKR map to those rigged configurations having configuration $\lambda$. However is there any, hopefully "good physical" meaning of these paths ?

This is a point where one can develop a further insight into fermionic formulas in the light of the soliton cellular automaton known as the box-ball system [7]. According to such studies initiated in [8] and accomplished in Theorem 7 for $s l_{2}$ case [9], those paths are characterized by the conserved quantities or equivalently by the soliton content $\lambda$. The $X(\lambda)$ thereby acquires the meaning of the partition function of the level set of the box-ball system. These features may be schematically stated as "solitons = strings", where the both sides can be defined mathematically within the framework of the box-ball system and the rigged configuration, respectively.

\subsection{Comparison with earlier results and layout of the paper}

The story (Theorem [7) in the previous subsection concerns the string hypothesis in the XXX Heisenberg spin chain. The relevant box-ball system is the one on the infinite lattice [7] truncated to the finite segment $[1, L]=\{j \in \mathbb{Z} \mid 1 \leq j \leq L\}$.

Our formula (11) provides an extension of such results in which XXX and the box-ball system are replaced with XXZ and the periodic box-ball system [10, 11, respectively. More specifically, the right hand side of (1) with $q=1$ precisely reproduces the counting formula of the Bethe roots for the XXZ Hamiltonian $\sum_{j \in \mathbb{Z}_{L}}\left(\sigma_{j}^{x} \sigma_{j+1}^{x}+\sigma_{j}^{y} \sigma_{j+1}^{y}+\Delta \sigma_{j}^{z} \sigma_{j+1}^{z}\right)$ at $\Delta=\infty[12$, eq. (3.11)]. With regard to the periodic box-ball system, it is defined on the ring $\mathbb{Z}_{L}$, and the level set $P_{L, \lambda}^{\circ}$ is equivalent to that introduced in [11], where the first complete solution of the initial value problem was obtained. A bold summary of these aspects is given in the following tables.

\begin{tabular}{c|c|c} 
string hypothesis & box-ball system & fermionic form \\
\hline $\operatorname{XXX}(\Delta=1)$ & {$[1, L]$} & $\mathcal{M}(\lambda, L), \mathcal{M}^{+}(\lambda, L) \quad$ eq. (9) \\
\hline $\operatorname{XXZ}(\Delta=\infty)$ & $\mathbb{Z}_{L}$ & $\mathcal{M}^{\circ}(\lambda, L) \quad$ eq. (16)
\end{tabular}

Compared with the fermionic forms $\mathcal{M}(\lambda, L)$ and $\mathcal{M}^{+}(\lambda, L)$, the new one $\mathcal{M}^{\circ}(\lambda, L)$ (= right hand side of eq. (11) ) takes the contributions of the solitons wrapping around the spurious boundary of the segment $[1, L]$. Compare Examples 5 and [14. Coping with them systematically and factorizing the whole level set partition function into a product of $q$-binomial coefficients require some technical analysis. It will be done in sections 4 and 5. Section 2 recalls the previous result (second line of the table) for comparison and convenience of the readers. In section 3 we describe the basic feature of the periodic box-ball system and formulate the main result of the paper in Theorem 16.

$\S$ A conceptual explanation of the correspondence between these values of $\Delta$ and the boundary conditions of the box-ball systems is yet to be found. 


\section{Fermionic expressions for one-dimensional configuration sums over non-periodic paths}

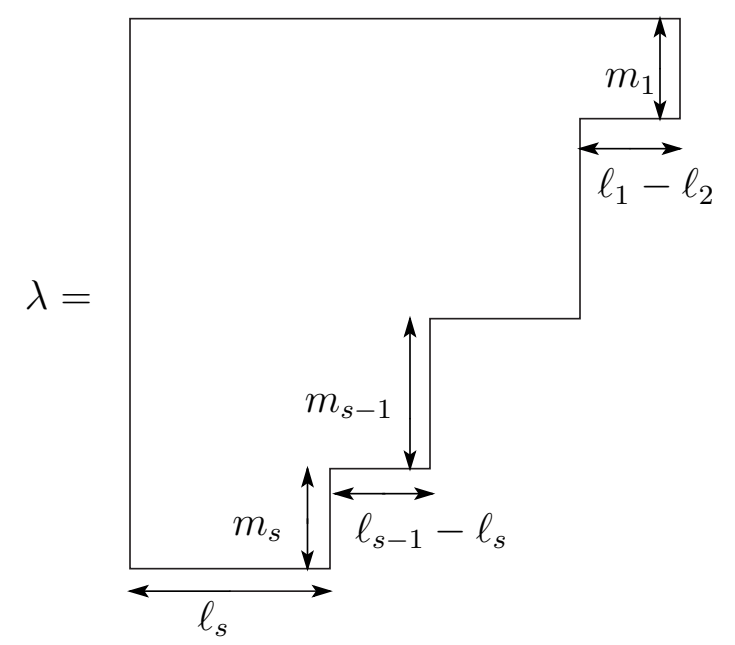

Figure 1. The Young diagram.

Fix any Young diagram $\lambda$. Suppose it has $m_{i}$ rows of length $\ell_{i}$ for $1 \leq i \leq s$, and $\ell_{1}>\ldots>\ell_{s}$. See Figure 1, We define

$$
\begin{aligned}
& p_{i}(\lambda):=L-2 \sum_{j=1}^{s} \min \left(\ell_{i}, \ell_{j}\right) m_{j}, \\
& \psi(\lambda):=\sum_{i, j=1}^{s} \min \left(\ell_{i}, \ell_{j}\right) m_{i} m_{j}-\sum_{i=1}^{s} \ell_{i} m_{i},
\end{aligned}
$$

which depend on $\lambda$ and a fixed integer $L$ satisfying $L \geq 2 \sum_{j=1}^{s} \ell_{j} m_{j}$. The $p_{i}=p_{i}(\lambda)$ 's are called vacancy numbers.

A Young diagram $\lambda$ is also regarded as a partition $\lambda=\left(\lambda_{1} \lambda_{2} \ldots\right)$ where $\lambda_{i}$ is called a part and equals to the length of the $i$-th row of $\lambda$. Parts are obeying the condition $\lambda_{1} \geq \lambda_{2} \geq \ldots \geq 0$ and those parts satisfying $\lambda_{i}=0$ are not explicitly written. If $|\lambda|:=\sum_{i} \lambda_{i}=M$ then $\lambda$ is call a partition of $M$ and denoted by $\lambda \vdash M$. We say that there are $\lambda_{i}$ boxes in the $i$-th row of the Young diagram $\lambda$.

By using this terminology, the $\psi(\lambda)$ is also calculated as follows. Fill 0's, 2's, 4's ... into the boxes of the first, second, third, ... rows of the Young diagram $\lambda$. Then the sum of these integers gives $\psi(\lambda)$.

Example 1 There are three partitions of 3, which are (3),(111) and (21). For these partitions, $\psi((3))=0+0+0=0, \psi((111))=0+2+4=6$ and $\psi((21))=0+0+2=2$.

Let $\boldsymbol{x}_{i}=\left(x_{i, j}\right)_{1 \leq j \leq m_{i}}, x_{i, j} \in \mathbb{Z}$ be a set of integers and set $\boldsymbol{x}=\left(\boldsymbol{x}_{i}\right)_{1 \leq i \leq s}$. The integers $x_{i, j}$ are called quantum number $\|$ or riggings. We set

$$
\Omega(\lambda, L)=\left\{\boldsymbol{x} \in \mathbb{Z}^{\mathcal{N}} \mid 0 \leq x_{i, 1} \leq \cdots \leq x_{i, m_{i}} \leq \ell_{i}+p_{i} \quad \text { for } \quad 1 \leq i \leq s\right\}
$$

$\|$ Conventionally the quantum numbers are given by $x_{i, j}-\ell_{i}$. 
Fermionic partition functions for a periodic soliton cellular automaton

$$
\Omega^{+}(\lambda, L)=\left\{\boldsymbol{x} \in \mathbb{Z}^{\mathcal{N}} \mid \ell_{i} \leq x_{i, 1} \leq \cdots \leq x_{i, m_{i}} \leq \ell_{i}+p_{i} \quad \text { for } \quad 1 \leq i \leq s\right\}
$$

where $\mathcal{N}=m_{1}+\cdots+m_{s}$.

Definition 2 (Energy of rigged configuration) For any pair $(\lambda, \boldsymbol{x})$ the quantity $E_{\mathrm{RC}}(\lambda, \boldsymbol{x}):=\psi(\lambda)+\sum_{i=1}^{s} \sum_{j=1}^{m_{i}} x_{i, j}$ is called its energy.

Let

$$
\begin{aligned}
\mathcal{M}(\lambda, L) & :=\sum_{\boldsymbol{x} \in \Omega(\lambda, L)} q^{E_{\mathrm{RC}}(\lambda, \boldsymbol{x})}, \\
\mathcal{M}^{+}(\lambda, L) & :=\sum_{\boldsymbol{x} \in \Omega^{+}(\lambda, L)} q^{E_{\mathrm{RC}}(\lambda, \boldsymbol{x})} .
\end{aligned}
$$

For any integers $n, m(n \geq m \geq 0)$ we use the notation:

$$
[n]=\frac{1-q^{n}}{1-q}, \quad\left[\begin{array}{c}
n \\
m
\end{array}\right]=\frac{(q)_{n}}{(q)_{m}(q)_{n-m}} \quad(q \text {-binomial coefficient }),
$$

where $(q)_{n}=(1-q)\left(1-q^{2}\right) \cdots\left(1-q^{n}\right)$. It is easy to see $\left[\begin{array}{c}p+m \\ m\end{array}\right]=\sum_{\mu} q^{|\mu|}$ where the sum is taken over all partitions $\mu$ which has at most $m$ parts and the largest part $\leq p$ [13, 14]. Hence we have:

Lemma 3 The following relations hold,

$$
\mathcal{M}(\lambda, L)=q^{\psi(\lambda)} \prod_{i=1}^{s}\left[\begin{array}{c}
p_{i}+m_{i}+\ell_{i} \\
m_{i}
\end{array}\right], \quad \mathcal{M}^{+}(\lambda, L)=q^{\psi(\lambda)+|\lambda|} \prod_{i=1}^{s}\left[\begin{array}{c}
p_{i}+m_{i} \\
m_{i}
\end{array}\right]
$$

Let $p=b_{1} \ldots b_{L}$ be a sequence of letters 1 and 2 where the number of 1 's is not less than that of 2's. Any such sequence is called a path. We emphasize that the above condition on the numbers of the letters 1 and 2 are always imposed throughout this paper for any path. If $b_{1} \ldots b_{i}$ is a path for any $1 \leq i \leq L$, then $p=b_{1} \ldots b_{L}$ is called a highest path. Let $P_{L, M}$ be the set of all paths of length $L$ with $M(\leq L / 2) 2$ 's

$$
P_{L, M}=\left\{b_{1} \ldots b_{L} \mid b_{i} \in\{1,2\}, \#\left\{i \mid b_{i}=2\right\}=M\right\},
$$

and $P_{L, M}^{+}$be the set of all highest paths $\subset P_{L, M}$.

Definition 4 (Energy of path) For any path $p=b_{1} \ldots b_{L} \in P_{L, M}$ the quantity $E_{\text {path }}(p):=\sum_{j=1}^{L-1} j \theta\left(b_{j}<b_{j+1}\right)$ is called its energy, where $\theta($ true $)=1, \theta($ false $)=0$.

The following results are well known

$$
\sum_{p \in P_{L, M}} q^{E_{\mathrm{path}}(p)}=\left[\begin{array}{c}
L \\
M
\end{array}\right], \quad \sum_{p \in P_{L, M}^{+}} q^{E_{\mathrm{path}}(p)}=\left[\begin{array}{c}
L \\
M
\end{array}\right]-\left[\begin{array}{c}
L \\
M-1
\end{array}\right] .
$$

Let $P_{L, \lambda}=\Psi^{-1}(\{\lambda\} \times \Omega(\lambda, L))$ and $P_{L, \lambda}^{+}=\Psi^{-1}\left(\{\lambda\} \times \Omega^{+}(\lambda, L)\right)$, where $\Psi$ is a bijective map to be defined in section 4.5 . 
Fermionic partition functions for a periodic soliton cellular automaton

Example 5 Let $L=6,|\lambda|=3$. Then

$$
\begin{aligned}
P_{6,(3)}= & \{222111,122211,112221,111222\}, \\
P_{6,(111)}= & \{212121,212112,211212,121212\}, \\
P_{6,(21)}= & \{212211,221211,211221,221121, \\
& 121221,211122,221112,122121, \\
& 121122,122112,112122,112212\} .
\end{aligned}
$$

By Theorem 26] we have $\bigsqcup_{\lambda \vdash M} P_{L, \lambda}=P_{L, M}$ and $\bigsqcup_{\lambda \vdash M} P_{L, \lambda}^{+}=P_{L, M}^{+}$. We define

$$
\begin{aligned}
X(\lambda, L) & :=\sum_{p \in P_{L, \lambda}} q^{E_{\mathrm{path}}(p)}, \\
X^{+}(\lambda, L) & :=\sum_{p \in P_{L, \lambda}^{+}} q^{E_{\mathrm{path}}(p)} .
\end{aligned}
$$

Example 6 By Example 5 it is easy to check

$$
\begin{aligned}
& X((3), 6)=1+q+q^{2}+q^{3}, \\
& X((111), 6)=q^{6}+q^{7}+q^{8}+q^{9}, \\
& X((21), 6)=q^{2}+2 q^{3}+3 q^{4}+3 q^{5}+2 q^{6}+q^{7} .
\end{aligned}
$$

As a result of Theorems 26 and 27, we obtain:

\section{Theorem 7 ([9])}

$$
\begin{aligned}
& X(\lambda, L)=\mathcal{M}(\lambda, L), \\
& X^{+}(\lambda, L)=\mathcal{M}^{+}(\lambda, L) .
\end{aligned}
$$

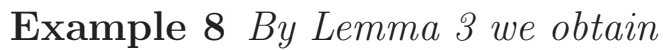

$$
\begin{aligned}
& \mathcal{M}((3), 6)=q^{0}\left[\begin{array}{c}
0+1+3 \\
1
\end{array}\right]=1+q+q^{2}+q^{3}, \\
& \mathcal{M}((111), 6)=q^{6}\left[\begin{array}{c}
0+3+1 \\
3
\end{array}\right]=q^{6}+q^{7}+q^{8}+q^{9}, \\
& \mathcal{M}((21), 6)=q^{2}\left[\begin{array}{c}
0+1+2 \\
1
\end{array}\right]\left[\begin{array}{c}
2+1+1 \\
1
\end{array}\right]=q^{2}+2 q^{3}+3 q^{4}+3 q^{5}+2 q^{6}+q^{7} .
\end{aligned}
$$

\section{A periodic soliton cellular automaton and its partition functions}

\subsection{The periodic box-ball system}

The periodic box-ball system (pBBS) is a one-dimensional cellular automaton with periodic boundary conditions. Denote by $\mathcal{P}=\sqcup_{M} P_{L, M}$ the set of all paths of length $L$. We can define a commuting family of time evolutions $T_{k}(k=1,2, \ldots)$ acting on $\mathcal{P}$.

Let $B_{k}$ be the set of all one-row semistandard tableaux of length $k$ with entries 1 and 2. For instance, $B_{1}=\{1,2\}, B_{2}=\{11,12,22\}$ and $B_{3}=\{111,112,122,222\}$. The 
combinatorial $R$ map $R: B_{k} \times B_{1} \rightarrow B_{1} \times B_{k}$ is defined as follows. Depict the relation $R(x, y)=(\tilde{y}, \tilde{x})$ by

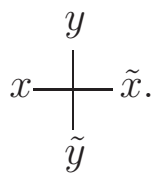

Then the definition of $R$ is given by the diagrams in Figure 2 .
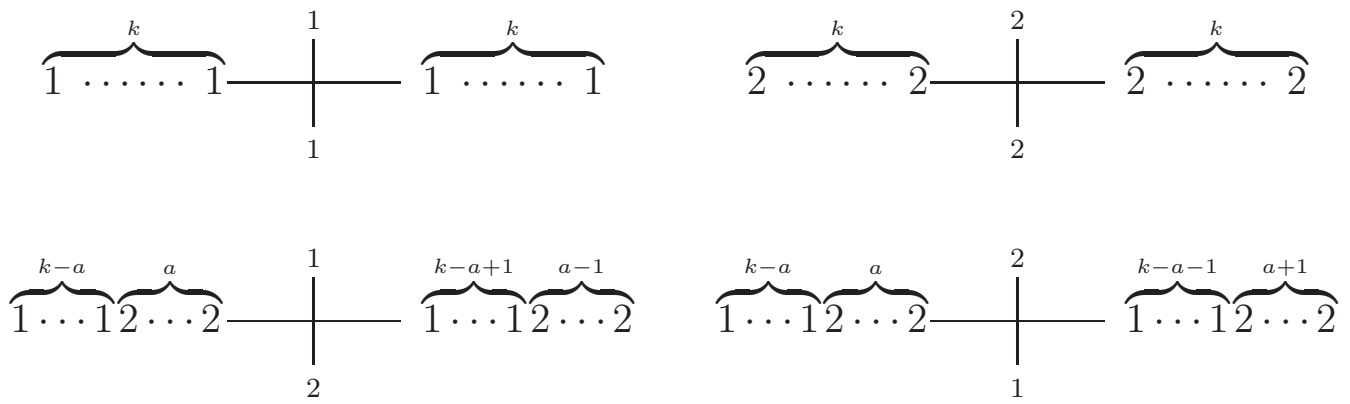

$(0<a \leq k)$

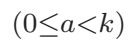

Figure 2. Combinatorial $R: B_{l} \times B_{1} \simeq B_{1} \times B_{l}$

By repeated use of this $R$ we define

$$
\begin{aligned}
& B_{k} \times\left(B_{1} \times \cdots \times B_{1}\right) \rightarrow\left(B_{1} \times \cdots \times B_{1}\right) \times B_{k} \\
& v \times\left(b_{1} \times \cdots \times b_{L}\right) \quad \mapsto\left(b_{1}^{\prime} \times \cdots \times b_{L}^{\prime}\right) \times v^{\prime} .
\end{aligned}
$$

Example 9 Set $k=3, L=13, v=112$ and $b_{1} \ldots b_{13}=1122121122211$. (We omit the symbol $\times$ here and in what follows.) Then we have $b_{1}^{\prime} \ldots b_{13}^{\prime}=2111212211122$ and $v^{\prime}=112$. It is verified as

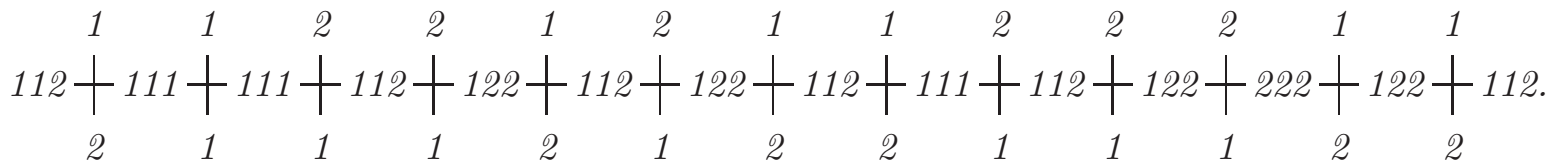

In this example we have $v=v^{\prime}$. In fact one can always find such $v, v^{\prime} \in B_{k}$ with this property.

Proposition 10 ([11]) Given any $b_{1} \times \cdots \times b_{L} \in \mathcal{P} \subset B_{1}^{\times L}$, let $v_{0} \in B_{k}$ be the one defined in the same way as (13) by

$$
\overbrace{1 \ldots 1}^{k} \times\left(b_{1} \times \cdots \times b_{L}\right) \mapsto\left(b_{1}^{\prime \prime} \times \cdots \times b_{L}^{\prime \prime}\right) \times v_{0} .
$$

Then we have $v=v^{\prime}$ in (13) when we adopt this $v_{0}$ as the $v$ there.

By this choice of $v$, we define the time evolution $T_{k}$ as $T_{k}\left(b_{1} \cdots b_{L}\right)=b_{1}^{\prime} \cdots b_{L}^{\prime}$ by (13).

Example 11 We have $T_{3}(1122121122211)=2111212211122$ by Example 9 . 
Here we give an example of the time evolution of this cellular automaton.

$$
\begin{aligned}
& t=0: 2221111111111122221112112211111 \\
& t=1: 1112221111111111112221221122111 \\
& t=2: 2111112221111111111112112211222 \\
& t=3: 1222211112221111111111211122111 \\
& t=4: 1111122221112221111111121111221 \\
& t=5: 2111111112221112222111112111112 \\
& t=6: 1221111111112221111222211211111 \\
& t=7: 1112211111111112221111122122211 \\
& t=8: 2211122111111111112221111211122 \\
& t=9: 1122211222111111111112221121111
\end{aligned}
$$

If we denote by $p \in \mathcal{P}$ the sequence for $t=0$ then the sequence for $t=n$ stands for $T_{4}^{n}(p) \in \mathcal{P}$.

When sufficiently separated from the other 2's, one can think of a consecutive sequence of 2's of length $k$ as a soliton of amplitude $k$. By an appropriate definition one can say that the number of solitons conserves for each amplitude. In this example there are four solitons of amplitudes 1,2,3 and 4 in every time step. In other words they have a common soliton content $\lambda=(4321)$.

A precise definition of the soliton content of a path $p=b_{1} \ldots b_{L}$ was presented in 11. Actually there are two equivalent (but apparently different) definitions, which we now recall quickly 1 . The first way of determining it is to consider the diagram as in Example 9 corresponding to the relation (13) with $v=v^{\prime}=v_{0} . \quad\left(v_{0}\right.$ is defined in Proposition [10.) Let $E_{k}=E_{k}(p)$ be the number of local vertices of the bottom right type in Figure 2 in the diagram. Then the soliton content is the Young diagram $\lambda$ such that $\lambda_{1}^{\prime}+\cdots+\lambda_{k}^{\prime}=E_{k}$, where $\lambda_{i}^{\prime}$ denotes the length of the $i$ th column of $\lambda$ from the left. The second way of finding the soliton content is to convert $p$ into a highest path $p^{\prime}$ by a cyclic shift and pick the configuration (=Young diagram $\lambda$ ) of $p^{\prime}$ under the KKR bijection [4, 5] from highest paths to rigged configurations. The result is unique even though $p^{\prime}$ is not necessarily so for a given $p$. It is known that the two definitions give the same $\lambda$ [11, Proposition 3.4]. The first and the second definitions have the meaning of solitons and strings respectively, therefore their coincidence is a manifestation of "solitons =strings" in the periodic box-ball system.

Given a fixed system size $L$, the set of all paths with a common soliton content is called a level set. We denote by $\mathcal{P}_{L, \lambda}^{\circ}$ the level set with soliton content $\lambda$. Then it is known [10, 11] that

$$
\left|\mathcal{P}_{L, \lambda}^{\circ}\right|=\frac{L}{p_{1}} \prod_{i=1}^{s}\left(\begin{array}{c}
p_{i}+m_{i}-1 \\
m_{i}
\end{array}\right) .
$$

In case $p_{1}=0$, the combination $\frac{L}{p_{1}}\left(\begin{array}{c}p_{1}+m_{1}-1 \\ m_{1}\end{array}\right)$ is to be understood as $\frac{L}{m_{1}}$.

I The definition based on the "arc rule" [10] also gives the equivalent result. 


\subsection{The partition function on a level set}

Let $\lambda$ be the Young diagram in Figure 1 and $L$ be an integer satisfying $L \geq 2 \sum_{j=1}^{s} \ell_{j} m_{j}$. In a similar way to (44) and (5) we set

$$
\Omega^{\circ}(\lambda, L)=\left\{\begin{array}{l|l}
\boldsymbol{x} \in \mathbb{Z}^{\mathcal{N}} & \begin{array}{l}
0 \leq x_{i, 1} \leq \cdots \leq x_{i, m_{i}} \leq p_{i}+\min \left(x_{i, 1}, 2 \ell_{i}-1\right) \\
\text { for } 1 \leq i \leq s, \\
x_{j, m_{j}}-p_{j} \leq x_{i, 1} \quad \text { for } 1 \leq i<j \leq s, \\
x_{i, m_{i}} \leq p_{i}+2\left(\ell_{i}-\ell_{j}\right)+x_{j, 1}-1 \text { for } 1 \leq i<j \leq s
\end{array}
\end{array}\right\} .
$$

Let

$$
\mathcal{M}^{\circ}(\lambda, L):=\sum_{\boldsymbol{x} \in \Omega^{\circ}(\lambda, L)} q^{E_{\mathrm{RC}}(\lambda, \boldsymbol{x})},
$$

where $E_{\mathrm{RC}}(\lambda, \boldsymbol{x})$ is given in Definition 2, In section 5 we will show:

Lemma 12 The following relation holds:

$$
\mathcal{M}^{\circ}(\lambda, L)=q^{\psi(\lambda)} \frac{[L]}{\left[p_{1}\right]} \prod_{i=1}^{s}\left[\begin{array}{c}
p_{i}+m_{i}-1 \\
m_{i}
\end{array}\right] \text {. }
$$

In case $p_{1}=0$, the combination $\frac{[L]}{\left[p_{1}\right]}\left[\begin{array}{c}p_{1}+m_{1}-1 \\ m_{1}\end{array}\right]$ is to be understood as $\frac{[L]}{\left[m_{1}\right]}$.

Let $P_{L, \lambda}^{\circ}=\Phi^{-1}\left(\{\lambda\} \times \Omega^{\circ}(\lambda, L)\right)$, where $\Phi$ is a bijective map to be defined in section 4.2. Hence $\left|P_{L, \lambda}^{\circ}\right|=\left|\{\lambda\} \times \Omega^{\circ}(\lambda, L)\right|=\left|\Omega^{\circ}(\lambda, L)\right|$. Note that RHS of (16) reduces to that of (14) when $q=1$, implying $\left|P_{L, \lambda}^{\circ}\right|=\left|\mathcal{P}_{L, \lambda}^{\circ}\right|$. In fact we have

Proposition $13 P_{L, \lambda}^{\circ}=\mathcal{P}_{L, \lambda}^{\circ}$.

Proof. This is due to the second definition of $P_{L, \lambda}^{\circ}$ based on the KKR bijection explained previously, the footnote on the quantum numbers in section 2 , Remark 22 and Theorem 4.3 of [15].

Example 14 Let $L=6,|\lambda|=3$. Then

$$
\begin{aligned}
P_{6,(3)}^{\circ}= & \{222111,122211,112221,111222,211122,221112\}, \\
P_{6,(111)}^{\circ}= & \{212121,121212\}, \\
P_{6,(21)}^{\circ}= & \{212211,221211,211221,221121, \\
& 121221,211212,212112,122121, \\
& 121122,122112,112122,112212\} .
\end{aligned}
$$

By Theorem 24 we have $\bigsqcup_{\lambda \vdash M} P_{L, \lambda}^{\circ}=P_{L, M}$. We define

$$
X^{\circ}(\lambda, L):=\sum_{p \in P_{L, \lambda}^{\circ}} q^{E_{\mathrm{path}}(p)} .
$$

Example 15 By Example 14 it is easy to check

$$
\begin{aligned}
& X^{\circ}((3), 6)=1+q+q^{2}+q^{3}+q^{4}+q^{5}, \\
& X^{\circ}((111), 6)=q^{6}+q^{9}, \\
& X^{\circ}((21), 6)=q^{2}+2 q^{3}+2 q^{4}+2 q^{5}+2 q^{6}+2 q^{7}+q^{8} .
\end{aligned}
$$


As a result of Theorems 24, 25 to be shown in subsection 4.4, and Lemma 12, we obtain the main result of this paper:

Theorem 16

$$
X^{\circ}(\lambda, L)=\mathcal{M}^{\circ}(\lambda, L) .
$$

Example 17 By Lemma 12 we obtain

$$
\begin{aligned}
& \mathcal{M}^{\circ}((3), 6)=q^{0} \frac{[6]}{[1]}=1+q+q^{2}+q^{3}+q^{4}+q^{5}, \\
& \mathcal{M}^{\circ}((111), 6)=q^{6} \frac{[6]}{[3]}=q^{6}+q^{9}, \\
& \mathcal{M}^{\circ}((21), 6)=q^{2} \frac{[6]}{[1]}\left[\begin{array}{c}
2+1-1 \\
1
\end{array}\right]=q^{2}+2 q^{3}+2 q^{4}+2 q^{5}+2 q^{6}+2 q^{7}+q^{8} .
\end{aligned}
$$

\section{A statistics preserving bijection between periodic paths and rigged configurations}

\subsection{Multisets}

We present an algorithm for a bijection between periodic paths and rigged configurations. The map from the former to the latter is referred to as a direct scattering transform, and its inverse is called an inverse scattering transform. To describe them, we first introduce the notion of multisets.

A multiset is a set with repeated elements which are assumed to be integers throughout this paper. When treating multisets, we respect the multiplicity of their elements. For instance $\{1,2,2,3\} \cap\{2,2,4,5\}$ is equal to $\{2,2\}$, not to $\{2\}$. And $\{1,2,2,3\} \backslash\{2,3\}$ is equal to $\{1,2\}$, not to $\{1\}$. Also we always rearrange elements of a multiset in weakly increasing order and call them its first, second, ..., last elements. For instance $\{1,2,2,3\} \cup\{2,2,4,5\}=\{1,2,2,2,2,3,4,5\}$, and its sixth element is 3 .

We define $\mathcal{O}_{0}, \mathcal{O}_{1}$ to be a pair of operators acting on multisets: Given any multiset $M$, the operator $\mathcal{O}_{0}$ (resp. $\left.\mathcal{O}_{1}\right)$ adds $2 i-2$ (resp. $2 i-1$ ) to its $i$-th element for all $i \in\{1, \ldots,|M|\}$. For instance, $\mathcal{O}_{0}(\{1,2,2,3\})=\{1,4,6,9\}$ and $\mathcal{O}_{1}(\{1,2,2,3\})=$ $\{2,5,7,10\}$. Their inverse operators $\mathcal{O}_{a}^{-1}(a=0,1)$ are defined by an obvious way.

\section{2. direct scattering transform}

The direct scattering transform is a map that sends a path $p=b_{1} \ldots b_{L}$ to the following data: a list of positive integer pairs $\lambda=\left\{\left(\ell_{1}, m_{1}\right), \ldots,\left(\ell_{s}, m_{s}\right)\right\}$ (soliton content) and an integer vector $\boldsymbol{x}=\left(\boldsymbol{x}_{i}\right)_{1 \leq i \leq s}, \boldsymbol{x}_{i}=\left(x_{i, j}\right)_{1 \leq j \leq m_{i}}$ obeying the condition $x_{i, 1} \leq \cdots \leq x_{i, m_{i}}$ (angle variables). Denote this map by $\Phi: p \mapsto(\lambda, \boldsymbol{x})$.

The path $p$ can be represented by a pair of multisets $M_{1}, M_{2}$ constructed by the following procedure: Let $M_{1}=\left\{0 \leq j \leq L \mid\left(b_{j}, b_{j+1}\right)=(1,2)\right\}$ and $M_{2}=$ $\left\{0 \leq j \leq L \mid\left(b_{j}, b_{j+1}\right)=(2,1)\right\}$ (arranged in weakly increasing order), where $b_{0}=b_{L+1}=1$. Since $\left|M_{1}\right|=\left|M_{2}\right|$ one can write them as $M_{1}=\left\{\alpha_{1}, \ldots, \alpha_{N}\right\}$ and 
$M_{2}=\left\{\beta_{1}, \ldots, \beta_{N}\right\}$ for some $N \in \mathbb{Z}_{>0}$. At the beginning their elements satisfy the conditions $0 \leq \alpha_{1}<\beta_{1}<\cdots<\alpha_{N}<\beta_{N} \leq L$ and $\left(\beta_{1}-\alpha_{1}\right)+\cdots+\left(\beta_{N}-\alpha_{N}\right) \leq L / 2$.

Let $M_{1}, M_{2}$ be the multisets for a path $p$ of length $L$. Introduce a pair of indices $j, k$, a list $D$, and a multiset $S$. To begin with we set $j=0, k=L, D=\{\}$ (an empty list), and $S=\emptyset$. Then run the following algorithm:

DS0 If $0 \in M_{1}$ and $L \in M_{2}$, then replace $M_{1}$ by $M_{1} \backslash\{0\}$ and $M_{2}$ by $\left(M_{2} \backslash\left\{\min M_{2}, L\right\}\right) \cup$ $\left\{\min M_{2}+L\right\}$.

DS1 While $M_{1} \cap M_{2}=\emptyset$ and $\max M_{2}-\min M_{1}<k$, continue replacing $\left(j, k, M_{1}, M_{2}\right)$ by $\left(j+1, k-2\left|M_{1}\right|, \mathcal{O}_{0}^{-1}\left(M_{1}\right), \mathcal{O}_{1}^{-1}\left(M_{2}\right)\right)$.

DS2 If $M_{1} \cap M_{2} \neq \emptyset$, then set $S=M_{1} \cap M_{2}$ and replace $\left(M_{1}, M_{2}\right)$ by $\left(M_{1} \backslash S, M_{2} \backslash S\right)$.

DS3 If $\max M_{2}-\min M_{1}=k$, then replace $S$ by $S \cup\left\{\min M_{1}\right\}, M_{1}$ by $M_{1} \backslash\left\{\min M_{1}\right\}$, and $M_{2}$ by $\left(M_{2} \backslash\left\{\min M_{2}, \max M_{2}\right\}\right) \cup\left\{\min M_{2}+k\right\}$.

DS4 Pre-pend (i.e. append as the first element) $\{S, j, k\}$ to $D$ and set $S=\emptyset$. If $M_{1}=\emptyset$ then stop. Otherwise go to step DS1.

At the end we obtain such type of data $D=\left\{\left\{S_{1}, j_{1}, k_{1}\right\},\left\{S_{2}, j_{2}, k_{2}\right\}, \ldots,\left\{S_{s}, j_{s}, k_{s}\right\}\right\}$ for some $s$, where $S_{1}, \ldots, S_{s}$ are multisets, $j_{1}, \ldots, j_{s}, k_{2}, \ldots, k_{s}$ are positive integers, and $k_{1}$ is a non-negative integer.

Let $\ell_{i}=j_{i}, p_{i}=k_{i}, m_{i}=\left|S_{i}\right|$ and $\boldsymbol{x}_{i}=S_{i}$.

We show that this algorithm is well-defined and reversible, as well as that the condition $\ell_{1}>\ldots>\ell_{s}>0$ holds. The reverse algorithm ( IS1 - IS4) will be given in the next subsection. Write the $M_{1}, M_{2}$ as $M_{1}=\left\{\alpha_{1}, \ldots, \alpha_{N}\right\}$ and $M_{2}=\left\{\beta_{1}, \ldots, \beta_{N}\right\}$ although their elements and even their cardinality $N$ will be repeatedly updated while the algorithm is running. Note that the following inequity is always satisfied:

$$
\left(\beta_{1}-\alpha_{1}\right)+\cdots+\left(\beta_{N}-\alpha_{N}\right) \leq k / 2 .
$$

About step DSo: When $\alpha_{1}=0$ and $\beta_{N}=L$ we redefine $M_{1}, M_{2}$ as $M_{1}=$ $\left\{\alpha_{2}, \ldots, \alpha_{N}\right\}, M_{2}=\left\{\beta_{2}, \ldots, \beta_{N-1}, \beta_{1}+L\right\}$. One has max $M_{2}>L$ when this action has been taken, while otherwise $\max M_{2} \leq L$. This implies that we can retrieve original $M_{1}, M_{2}$ by taking step IS4 in the reverse algorithm.

About step $\boldsymbol{D S 1}$ : Every replacement in step DS1 reduces $\beta_{i}-\alpha_{i}, \alpha_{i+1}-\beta_{i}$ and $k-\left(\beta_{N}-\alpha_{1}\right)$ by one. Thus $M_{1} \cap M_{2} \neq \emptyset$ or $\max M_{2}-\min M_{1}=k$ will be certainly attained after taking this action finite times. Since no rearrangements of elements occur during this step, one can go backwards and retrieve original $M_{1}, M_{2}$ from their final expressions in this step.

About steps $\mathbf{D S 2}$ and $\boldsymbol{D S} 3$ :

A reverse procedure for these steps is given by IS1 and IS2 in the algorithm of the inverse scattering transform. Suppose step DS3 is relevant. (The other case is easier. 
If DS3 is irrelevant, then so is IS2 in the reverse algorithm. It will be also shown that there is a condition on the expressions of the $M_{1}$ and $M_{2}$ just after DS3 which enables us to distinguish whether step DS3 has been relevant or not.) Then the $M_{1}, M_{2}$ which one has just before step DS2 can be written as

$$
M_{1}=\left\{\alpha_{1}, \ldots, \alpha_{N}\right\}, M_{2}=\left\{\beta_{1}, \ldots, \beta_{N}=\alpha_{1}+k\right\} .
$$

In order to leave an $\alpha_{1}$ in $M_{1}$ just after step DS2 there must be odd number of $\alpha_{1}$ 's in $M_{1} \cup M_{2}$. Thus one has

$$
\begin{aligned}
& \alpha_{1}=\beta_{1}=\cdots=\alpha_{p-1}=\beta_{p-1}=\alpha_{p}<\beta_{p}<\alpha_{p+1}, \quad \text { or } \\
& \alpha_{1}=\beta_{1}=\cdots=\alpha_{p-1}=\beta_{p-1}=\alpha_{p}<\beta_{p}=\alpha_{p+1} \leq \cdots \leq \beta_{q-1}=\alpha_{q} \leq \beta_{q}<\alpha_{q+1},
\end{aligned}
$$

for some $p, q(1 \leq p<q)$. Consider the latter case. (The former case is easier.) Write the $M_{1}, M_{2}$ which one has just after step DS2 as $M_{1}=\left\{\alpha_{1}^{\prime}, \ldots, \alpha_{M}^{\prime}\right\}$ and $M_{2}=\left\{\beta_{1}^{\prime}, \ldots, \beta_{M}^{\prime}\right\}$ where $\alpha_{1}^{\prime}=\alpha_{1}, \beta_{1}^{\prime}=\beta_{q}$, and $\beta_{M}^{\prime}=\alpha_{1}+k$. Assuming $M=1$ one has $\beta_{1}^{\prime}-\alpha_{1}^{\prime}=k>k / 2$, which contradicts (18). Hence $M \geq 2$. Now we get

$$
M_{1}=\left\{\alpha_{2}^{\prime}, \ldots, \alpha_{M}^{\prime}\right\}, M_{2}=\left\{\beta_{2}^{\prime}, \ldots, \beta_{M-1}^{\prime}, \beta_{q}+k\right\},
$$

and

$$
S=\{\overbrace{\alpha_{1}, \ldots, \alpha_{1}}^{p}, \alpha_{p+1}, \ldots, \alpha_{q}, \ldots\},
$$

just after step DS3. Note that $\alpha_{2}^{\prime} \geq \alpha_{q+1}$.

Now let us consider how one can retrieve the original $M_{1}, M_{2}$. Starting from (20) and (21), we replace $\left(M_{1}, M_{2}\right)$ by $\left(M_{1} \cup S, M_{2} \cup S\right)$ according to IS1. Then

$$
\begin{aligned}
& M_{1}=\{\overbrace{\alpha_{1}, \ldots, \alpha_{1}}^{p}, \alpha_{p+1}, \ldots, \alpha_{q}, \alpha_{q+1}, \ldots, \alpha_{N-1}, \alpha_{N}\}, \\
& M_{2}=\{\overbrace{\alpha_{1}, \ldots, \alpha_{1}}^{p}, \alpha_{p+1}, \ldots, \alpha_{q}, \beta_{q+1}, \ldots, \beta_{N-1}, \beta_{q}+k\} .
\end{aligned}
$$

Hence the $M_{1}$ in (19) has been retrieved. Then applying IS2 we get the $M_{2}$ in (19) by replacing $M_{2}$ by $\left(M_{2} \backslash\left\{\min M_{2}, \max M_{2}\right\}\right) \cup\left\{\max M_{2}-k, \min M_{2}+k\right\}$. Note that $\max M_{2}-\min M_{1}>k$ is satisfied for (22), (23), while if DS3 is irrelevant this does not hold. This is the condition that we have mentioned just above (19).

About the condition $\ell_{1}>\ldots>\ell_{s}>0$ :

We note that for (20) one has $\max M_{2}-\min M_{1}=\beta_{q}+k-\alpha_{2}^{\prime} \leq \beta_{q}+k-\alpha_{q+1}<k$. Hence whether step DS3 is relevant or not, we have $M_{1} \cap M_{2}=\emptyset$ and $\max M_{2}-\min M_{1}<$ $k$ just after step DS3. These conditions are also valid just after step DS0. Thus the increment $j \rightarrow j+1$ in the procedure in step DS1 is certainly done at least once whenever the algorithm takes this step, leading to the condition $\ell_{1}>\ldots>\ell_{s}>0$.

Example 18 Consider the path $p=21221221111221$ of length $L=14$. Then $M_{1}=\{0,2,5,11\}$ and $M_{2}=\{1,4,7,13\}$. By applying the procedure in step $\boldsymbol{D} \boldsymbol{S} \mathbf{1}$ once, we obtain $j=1, k=14-8=6, M_{1}=\{0,0,1,5\}$ and $M_{2}=\{0,1,2,6\}$. By DS2 we set $S=\{0,1\}$ and redefine $M_{1}$ as $M_{1}=\{0,5\}, M_{2}$ as $M_{2}=\{2,6\}$. By DS3 we set 
$S=\{0,0,1\}$ and redefine $M_{1}$ as $M_{1}=\{5\}, M_{2}$ as $M_{2}=\{8\}$. Then by $\boldsymbol{D S} 4$ we have $D=\{\{\{0,0,1\}, 1,6\}\}$, and go to $\boldsymbol{D S} \mathbf{1}$.

In the second turn, we obtain $j=4, k=6-3 \times 2=0, M_{1}=M_{2}=\{5\}$ after applying the procedure in $\boldsymbol{D S 1}$ three times. By $\boldsymbol{D S} 2$ we set $S=\{5\}$ and redefine $M_{1}$ and $M_{2}$ as $M_{1}=M_{2}=\emptyset$. Then by $\boldsymbol{D S} 4$ we have $D=\{\{\{5\}, 4,0\},\{\{0,0,1\}, 1,6\}\}$, and stop the algorithm.

Hence we have $\left(\ell_{1}, \ell_{2}\right)=(4,1),\left(p_{1}, p_{2}\right)=(0,6),\left(m_{1}, m_{2}\right)=(1,3)$ and $\boldsymbol{x}=$ $\left(x_{1,1}, x_{2,1}, x_{2,2}, x_{2,3}\right)=(5,0,0,1)$.

Example 19 Consider the path $p=22211222111111221$ of length $L=17$. Then $M_{1}=\{0,5,14\}$ and $M_{2}=\{3,8,16\}$. By applying the procedure in DS1 once, we obtain $j=1, k=17-6=11, M_{1}=\{0,3,10\}$ and $M_{2}=\{2,5,11\}$. By DS3 we set $S=\{0\}$ and redefine $M_{1}$ as $M_{1}=\{3,10\}, M_{2}$ as $M_{2}=\{5,13\}$. Then by $\boldsymbol{D S} 4$ we have $D=\{\{\{0\}, 1,11\}\}$, and go to $\boldsymbol{D S} \mathbf{1}$.

In the second turn, we obtain $j=2, k=11-4=7, M_{1}=\{3,8\}$ and $M_{2}=\{4,10\}$ after applying the procedure in $\boldsymbol{D S} \boldsymbol{1}$ once. By $\boldsymbol{D S} 3$ we set $S=\{3\}$ and redefine $M_{1}$ as $M_{1}=\{8\}, M_{2}$ as $M_{2}=\{11\}$. Then by $\mathbf{D S} 4$ we have $D=\{\{\{3\}, 2,7\},\{\{0\}, 1,11\}\}$, and go to $\mathbf{D S 1}$.

In the third turn, we obtain $j=5, k=7-3 \times 2=1, M_{1}=M_{2}=\{8\}$ after applying the procedure in $\mathbf{D S 1}$ three times. Then by $\mathbf{D S 2}$ and $\mathbf{D S} 4$ we have $D=\{\{\{8\}, 5,1\},\{\{3\}, 2,7\},\{\{0\}, 1,11\}\}, M_{1}=M_{2}=\emptyset$, and stop the algorithm.

Hence we have $\left(\ell_{1}, \ell_{2}, \ell_{3}\right)=(5,2,1),\left(p_{1}, p_{2}, p_{3}\right)=(1,7,11),\left(m_{1}, m_{2}, m_{3}\right)=(1,1,1)$ and $\boldsymbol{x}=\left(x_{1,1}, x_{2,1}, x_{3,1}\right)=(8,3,0)$.

Example 20 Consider the path $p=22221111222211111222111211122211122221$ of length $L=38$. Then $M_{1}=\{0,8,17,23,27,33\}$ and $M_{2}=\{4,12,20,24,30,37\}$. By applying the procedure in DS1 once, we obtain $j=1, k=38-12=26, M_{1}=$ $\{0,6,13,17,19,23\}$ and $M_{2}=\{3,9,15,17,21,26\}$. By DS2 we set $S=\{17\}$ and redefine $M_{1}$ as $M_{1}=\{0,6,13,19,23\}, M_{2}$ as $M_{2}=\{3,9,15,21,26\}$. By $\boldsymbol{D S}$ 3 we redefine $S$ as $S=\{0,17\}, M_{1}$ as $M_{1}=\{6,13,19,23\}, M_{2}$ as $M_{2}=\{9,15,21,29\}$. Then by $\boldsymbol{D S} 4$ we have $D=\{\{\{0,17\}, 1,26\}\}$, and go to $\boldsymbol{D S} \mathbf{1}$.

In the second turn, we obtain $j=3, k=26-2 \times 8=10, M_{1}=\{6,9,11,11\}$ and $M_{2}=\{7,9,11,15\}$ after applying the procedure in $\boldsymbol{D S} \boldsymbol{1}$ twice. Then by $\boldsymbol{D S} \boldsymbol{2}$ and $\boldsymbol{D S} 4$ one has $D=\{\{\{9,11\}, 3,10\},\{\{0,17\}, 1,26\}\}, M_{1}=\{6,11\}, M_{2}=\{7,15\}$, and go to DS1.

After two more turns, one has

$$
D=\{\{\{9\}, 7,0\},\{\{6\}, 4,6\},\{\{9,11\}, 3,10\},\{\{0,17\}, 1,26\}\},
$$

$M_{1}=M_{2}=\emptyset$, and stop the algorithm. Hence we have $\left(\ell_{1}, \ell_{2}, \ell_{3}, \ell_{4}\right)=$ $(7,4,3,1),\left(p_{1}, p_{2}, p_{3}, p_{4}\right)=(0,6,10,26),\left(m_{1}, m_{2}, m_{3}, m_{4}\right)=(1,1,2,2)$ and

$$
\boldsymbol{x}=\left(x_{1,1}, x_{2,1}, x_{3,1}, x_{3,2}, x_{4,1}, x_{4,2}\right)=(9,6,9,11,0,17) .
$$

Remark 21 By construction the relations $p_{1}=L-2 \sum_{i=1}^{s} \ell_{i} m_{i}$ and $p_{i}=p_{i-1}+2\left(m_{1}+\right.$ $\left.\cdots+m_{i-1}\right)\left(\ell_{i-1}-\ell_{i}\right) \quad(2 \leq i \leq s)$ hold. Thus the calculation of the vacancy numbers 
Fermionic partition functions for a periodic soliton cellular automaton

in the algorithm is optional. We assumed that the number of 1 's is not less than that of 2 's for any paths, which implies $p_{1} \geq 0$. In addition, the condition $\ell_{1}>\ldots>\ell_{s}>0$ holds as we mentioned. Thus we have $0 \leq p_{1}<\cdots<p_{s}(<L)$.

Remark 22 Our $\Phi$ is equivalent to the map introduced in [16] under the name of '10elimination'. The elements of $\boldsymbol{x}$ in this paper coincide with the data called 'the positions of 0-solitons' in [16]. In terms of the 10-elimination, the vacancy numbers represent effective sizes of the system.

\subsection{Inverse scattering transform}

The inverse scattering transform is defined as the inverse of $\Phi$ that sends soliton content $\lambda=\left\{\left(\ell_{1}, m_{1}\right), \ldots,\left(\ell_{s}, m_{s}\right)\right\}$ and angle variable $\boldsymbol{x}=\left(\boldsymbol{x}_{i}\right)_{1 \leq i \leq s}$ to a path $p$. Denote this map by $\Phi^{-1}:(\lambda, \boldsymbol{x}) \mapsto p$.

Let $S_{i}=\boldsymbol{x}_{i}$ interpreted as a multiset, and $p_{i}=p_{i}(\lambda)$ be the vacancy number. Essentially, the inverse scattering transform is a map that sends data $D=\left\{\left\{S_{1}, \ell_{1}, p_{1}\right\},\left\{S_{2}, \ell_{2}, p_{2}\right\}, \ldots,\left\{S_{s}, \ell_{s}, p_{s}\right\}\right\}$ to a pair of multisets $M_{1}, M_{2}$. Now we describe it based on the discussion on the reversibility of $\Phi$ in the previous subsection.

To begin with we set $M_{1}=M_{2}=\emptyset$ and $i=1$. Then run the following algorithm.

IS1 Replace $M_{1}$ by $M_{1} \cup S_{i}$ and $M_{2}$ by $M_{2} \cup S_{i}$.

IS2 If $\max M_{2}-\min M_{1}>p_{i}$, then replace $M_{2}$ by $\left(M_{2} \backslash\left\{\min M_{2}, \max M_{2}\right\}\right) \cup\left\{\max M_{2}-\right.$ $\left.p_{i}, \min M_{2}+p_{i}\right\}$.

IS3 Replace $M_{1}$ by $\mathcal{O}_{0}^{\ell_{i}-\ell_{i+1}}\left(M_{1}\right)$ and $M_{2}$ by $\mathcal{O}_{1}^{\ell_{i}-\ell_{i+1}}\left(M_{2}\right)$. If $i<s$, then replace $i$ by $i+1$ and go back to step IS1. If $i=s$, then go to step IS4 where we interpret $\ell_{s+1}=0$.

IS4 If $\max M_{2}>L$, then replace $M_{1}$ by $M_{1} \cup\{0\}$ and $M_{2}$ by $\left(M_{2} \backslash\left\{\max M_{2}\right\}\right) \cup$ $\left\{\max M_{2}-L, L\right\}$.

Path $p=b_{1} \cdots b_{L} \in P_{L, M}$ can be represented by multisets $M_{1}=\left\{\alpha_{1}, \ldots, \alpha_{N}\right\}$ and $M_{2}=\left\{\beta_{1}, \ldots, \beta_{N}\right\}$ for some $N$. Their elements obey the following conditions:

$$
\begin{aligned}
& 0 \leq \alpha_{1}<\beta_{1}<\cdots<\alpha_{N}<\beta_{N} \leq L, \\
& \sum_{i=1}^{N}\left(\beta_{i}-\alpha_{i}\right)=M
\end{aligned}
$$

To obtain $p \in P_{L, M}$ from $M_{1}, M_{2}$, set $b_{i}=1$ (resp. $b_{i}=2$ ) if $\beta_{k-1}<i \leq \alpha_{k}$ (resp. $\alpha_{k}<i \leq \beta_{k}$ ) for some $k$. Here we interpret $\beta_{-1}=0$ and $\alpha_{N+1}=L$. Since $\Phi$ is reversible, it gives a bijection between $P_{L, M}$ and $\Phi\left(P_{L, M}\right)$. In the next subsection we classify the elements of $\Phi\left(P_{L, M}\right)$ according to their soliton contents. For this purpose we establish a lemma in this subsection.

Let $\lambda=\left\{\left(\ell_{i}, m_{i}\right)\right\}_{1 \leq i \leq s}$ be a soliton content and recall the set $\Omega^{\circ}(\lambda, L)$ defined in subsection 3.2 where the angle variables $\boldsymbol{x}=\left(\boldsymbol{x}_{i}\right)_{1 \leq i \leq s}, \boldsymbol{x}_{i}=\left(x_{i, j}\right)_{1 \leq j \leq m_{i}}$ are satisfying 
the conditions:

$$
\begin{aligned}
& 0 \leq x_{i, 1} \leq \cdots \leq x_{i, m_{i}} \text { for } 1 \leq i \leq s, \\
& x_{i, m_{i}} \leq p_{i}+\min \left(x_{i, 1}, 2 \ell_{i}-1\right) \text { for } 1 \leq i \leq s, \\
& x_{j, m_{j}}-p_{j} \leq x_{i, 1} \text { for } 1 \leq i<j \leq s, \\
& x_{i, m_{i}} \leq p_{i}+2\left(\ell_{i}-\ell_{j}\right)+x_{j, 1}-1 \text { for } 1 \leq i<j \leq s .
\end{aligned}
$$

Lemma 23 For any $\lambda \vdash M$, the condition $\{\lambda\} \times \Omega^{\circ}(\lambda, L) \subset \Phi\left(P_{L, M}\right)$ holds.

Proof. Given $\lambda \vdash M$ and $\boldsymbol{x} \in \Omega^{\circ}(\lambda, L)$, let $D$ be the data associated with $(\lambda, \boldsymbol{x})$. We are to show that the algorithm of $\Phi^{-1}$ is well-defined for such $D$, and the resulting $M_{1}, M_{2}$ satisfy the conditions (24), (25).

Recall the multisets $M_{1}, M_{2}$ used in the algorithm. Write them as $M_{1}=$ $\left\{\alpha_{1}, \ldots, \alpha_{N}\right\}$ and $M_{2}=\left\{\beta_{1}, \ldots, \beta_{N}\right\}$ although their elements and even their cardinality $N$ will be repeatedly updated while the algorithm is running. It will be shown that the following conditions are satisfied:

(i) $\alpha_{1} \leq \beta_{1} \leq \cdots \leq \alpha_{N} \leq \beta_{N}$ and $\alpha_{N}-\alpha_{1} \leq p_{i}$, just after step IS1.

(ii) $\alpha_{1} \leq \beta_{1} \leq \cdots \leq \alpha_{N} \leq \beta_{N}$ and $\beta_{N}-\alpha_{1} \leq p_{i}$, just after step IS2.

(iii) $\alpha_{1}<\beta_{1}<\cdots<\alpha_{N}<\beta_{N}$ and $\beta_{N}-\alpha_{1}<p_{i+1}$, just after step IS3.

(iv) $0 \leq \alpha_{1}$ and $\alpha_{N} \leq L-1$, just before step IS4.

Here we interpret $p_{s+1}=p_{s}+2\left(m_{1}+\cdots+m_{s}\right) \ell_{s}=L$. We shall prove (i)-(iv) later. The statements of items (i)-(iii) are for the $i$-th turn of the algorithm. We denote by $(\mathrm{x})_{k}$ the statement of item (x) for the $k$-th turn. Items $(\mathrm{i})_{1},(\mathrm{ii})_{1}$, and (iv) can be proved independently. We shall show that: 1 . item $(\mathrm{i})_{k>1}$ holds under the assumption of item (iii) $)_{k-1} ; 2$. item $(\mathrm{ii})_{k>1}$ holds under (iii) $)_{k-1}$ and $(\mathrm{i})_{k} ; 3$. item (iii) $)_{k}$ holds under (ii) $)_{k}$. Thus $(\mathrm{i})_{k^{-}}(\mathrm{iii})_{k}$ can be proved by induction on $k$.

The Lemma follows from items (iii) and (iv). Denote those $M_{1}$ and $M_{2}$ that one has just before step IS4 by $M_{1}^{\prime}=\left\{\alpha_{1}^{\prime}, \ldots, \alpha_{N}^{\prime}\right\}$ and $M_{2}^{\prime}=\left\{\beta_{1}^{\prime}, \ldots, \beta_{N}^{\prime}\right\}$. Then we have $0 \leq \alpha_{1}^{\prime}<\beta_{1}^{\prime}<\ldots<\alpha_{N}^{\prime}<\beta_{N}^{\prime}, \beta_{N}^{\prime}-\alpha_{1}^{\prime}<p_{s+1}=L$ and $\alpha_{N}^{\prime} \leq L-1$ by items (iii) and (iv). We can also derive

$$
\sum_{i=1}^{N}\left(\beta_{i}^{\prime}-\alpha_{i}^{\prime}\right)=\sum_{i=1}^{s}\left(m_{1}+\cdots+m_{i}\right)\left(\ell_{i}-\ell_{i+1}\right)=\sum_{i=1}^{s} m_{i} \ell_{i}=|\lambda|,
$$

based on step IS3 where the relation $\left|M_{1}\right|=\left|M_{2}\right|=m_{1}+\cdots+m_{i}$ holds in the $i$-th turn.

We are to show that the $M_{1}, M_{2}$ that one has after step IS4 satisfy the conditions (24), (25) with $M=|\lambda|$. If $\beta_{N}^{\prime} \leq L$ then step IS4 is irrelevant and the Lemma follows immediately.

Suppose $\beta_{N}^{\prime}>L$ and write the $M_{1}, M_{2}$ that one has after step IS4 as $M_{1}=$ $\left\{\alpha_{1}, \ldots, \alpha_{N+1}\right\}, M_{2}=\left\{\beta_{1}, \ldots, \beta_{N+1}\right\}$. Since $M_{1}=M_{1}^{\prime} \cup\{0\}$ and $\alpha_{1}^{\prime} \geq 0$, we have $\alpha_{1}=0$ and $\alpha_{i}=\alpha_{i-1}^{\prime}(2 \leq i \leq N+1)$ for the elements of $M_{1}$. While the $M_{2}$ is given in terms of the elements of $M_{2}^{\prime}$ by $M_{2}=\left\{\beta_{1}^{\prime}, \ldots, \beta_{N-1}^{\prime}\right\} \cup\left\{\beta_{N}^{\prime}-L, L\right\}$. By assumption we 
have $\beta_{N}^{\prime}-L<\alpha_{1}^{\prime}<\beta_{1}^{\prime}$, and $L>\alpha_{N}^{\prime}>\beta_{N-1}^{\prime}$. Hence $\beta_{1}=\beta_{N}^{\prime}-L, \beta_{N+1}=L$, and $\beta_{i}=$ $\beta_{i-1}^{\prime}(2 \leq i \leq N)$. Now it is easy to see that the elements of $M_{1}, M_{2}$ obey the conditions $0=\alpha_{1}<\beta_{1}<\ldots<\alpha_{N+1}<\beta_{N+1}=L$ and $\sum_{i=1}^{N+1}\left(\beta_{i}-\alpha_{i}\right)=\sum_{i=1}^{N}\left(\beta_{i}^{\prime}-\alpha_{i}^{\prime}\right)=|\lambda|$.

Proof of item (i): When $i=1$ we have $M_{1}=M_{2}=S_{1}=\left\{x_{1,1}, \ldots, x_{1, m_{1}}\right\}$. Hence the claim follows by (26) and (27). Suppose $i>1$. Denote by $M_{1}^{\prime}=\left\{\alpha_{1}^{\prime}, \ldots, \alpha_{M}^{\prime}\right\}$ and $M_{2}^{\prime}=\left\{\beta_{1}^{\prime}, \ldots, \beta_{M}^{\prime}\right\}$ the $M_{1}, M_{2}$ that one has just after step IS3 in the $(i-1)$-th turn. We assume the conditions $\alpha_{1}^{\prime}<\beta_{1}^{\prime}<\ldots<\alpha_{M}^{\prime}<\beta_{M}^{\prime}$ and $\beta_{M}^{\prime}-\alpha_{1}^{\prime}<p_{i}$. Let $M_{1}=\left\{\alpha_{1}, \ldots, \alpha_{N}\right\}$ and $M_{2}=\left\{\beta_{1}, \ldots, \beta_{N}\right\}$ be the $M_{1}, M_{2}$ that one has just after step IS1 in the $i$-th turn. Then $M_{1}=M_{1}^{\prime} \cup S_{i}$ and $M_{2}=M_{2}^{\prime} \cup S_{i}$ with $S_{i}=\left\{x_{i, 1}, \ldots, x_{i, m_{i}}\right\}$. Clearly the condition $\alpha_{1} \leq \beta_{1} \leq \cdots \leq \alpha_{N} \leq \beta_{N}$ holds. Let us show $\alpha_{N}-\alpha_{1} \leq p_{i}$. It suffices to consider the following four cases:

case $1 \alpha_{1}=x_{i, 1}, \alpha_{N}=x_{i, m_{i}}$ : By (27) we have $\alpha_{N}-\alpha_{1}=x_{i, m_{i}}-x_{i, 1} \leq p_{i}$.

case $2 \alpha_{1}=\alpha_{1}^{\prime}, \alpha_{N}=\alpha_{M}^{\prime}$ : Since $\beta_{M}^{\prime}-\alpha_{1}^{\prime}<p_{i}$ and $\alpha_{M}^{\prime}<\beta_{M}^{\prime}$, we have $\alpha_{N}-\alpha_{1}=$ $\alpha_{M}^{\prime}-\alpha_{1}^{\prime}<\beta_{M}^{\prime}-\alpha_{1}^{\prime}<p_{i}$

case $3 \alpha_{1}=\alpha_{1}^{\prime}, \alpha_{N}=x_{i, m_{i}}$ : The $\alpha_{1}^{\prime}$ is given as $\alpha_{1}^{\prime}=x_{j, 1}$ for some $j(<i)$. Hence $\alpha_{N}-\alpha_{1}=x_{i, m_{i}}-x_{j, 1} \leq p_{i}$ by (28).

case $4 \alpha_{1}=x_{i, 1}, \alpha_{N}=\alpha_{M}^{\prime}$ : The $\alpha_{M}^{\prime}$ is given by the following formula for some $j(<i)$ :

$$
\begin{aligned}
\alpha_{M}^{\prime} & =x_{j, m_{j}}+2 \sum_{k=j}^{i-1}\left(\ell_{k}-\ell_{k+1}\right)\left(\sum_{h=1}^{k} m_{h}-1\right) \\
& =x_{j, m_{j}}+\sum_{k=j}^{i-1}\left(\left(p_{k+1}-p_{k}\right)-2\left(\ell_{k}-\ell_{k+1}\right)\right) \\
& =x_{j, m_{j}}+p_{i}-p_{j}+2 \ell_{i}-2 \ell_{j} .
\end{aligned}
$$

Hence $\alpha_{N}-\alpha_{1}=x_{j, m_{j}}-x_{i, 1}+p_{i}-p_{j}+2 \ell_{i}-2 \ell_{j}<p_{i}$ by (29).

Proof of item (ii): When $i=1$ step IS2 is irrelevant, hence $M_{1}=M_{2}=S_{1}=$ $\left\{x_{1,1}, \ldots, x_{1, m_{1}}\right\}$. Thus the claim follows by (26) and (27). Suppose $i>1$. Set $\alpha=\min M_{1}^{\prime \prime}$ and $\beta=\max M_{2}^{\prime \prime}$ where $M_{1}^{\prime \prime}, M_{2}^{\prime \prime}$ are the $M_{1}, M_{2}$ that one has just after step IS3 in the $(i-1)$-th turn. Assume $\beta-\alpha<p_{i}$. We denote by $M_{1}^{\prime}=\left\{\alpha_{1}^{\prime}, \ldots, \alpha_{N}^{\prime}\right\}$ and $M_{2}^{\prime}=\left\{\beta_{1}^{\prime}, \ldots, \beta_{N}^{\prime}\right\}$ the $M_{1}, M_{2}$ that one has just after step IS1 in the $i$-th turn, and assume that the conditions $\alpha_{1}^{\prime} \leq \beta_{1}^{\prime} \leq \ldots \leq \alpha_{N}^{\prime} \leq \beta_{N}^{\prime}$ and $\alpha_{N}^{\prime}-\alpha_{1}^{\prime} \leq p_{i}$ are satisfied. Let $M_{1}=\left\{\alpha_{1}, \ldots, \alpha_{N}\right\}$ and $M_{2}=\left\{\beta_{1}, \ldots, \beta_{N}\right\}$ be the $M_{1}, M_{2}$ that one has just after step IS2 in the $i$-th turn. Since IS2 keeps $M_{1}$ unchanged, one always has $\alpha_{k}=\alpha_{k}^{\prime}(1 \leq k \leq N)$. If $\beta_{N}^{\prime}-\alpha_{1}^{\prime} \leq p_{i}$ then $\beta_{k}=\beta_{k}^{\prime}(1 \leq k \leq N)$, hence follows item (ii).

Suppose $\beta_{N}^{\prime}-\alpha_{1}^{\prime}>p_{i}$. In this case $\beta_{N}^{\prime}=\beta$, because otherwise one has $\beta_{N}^{\prime}=x_{i, m_{i}}=\alpha_{N}^{\prime}$ and hence $\beta_{N}^{\prime}-\alpha_{1}^{\prime} \leq p_{i}$. Let $p$ be the smallest integer such that $\alpha_{p}^{\prime}=\alpha$. Then we have $p>1$, because otherwise one has $\beta_{N}^{\prime}-\alpha_{1}^{\prime}=\beta-\alpha<p_{i}$. By definition of IS1, one has $\alpha_{k}^{\prime}=\beta_{k}^{\prime}$ for all $k \in\{1 \ldots, p-1\}$. By definition of IS2, one 
has $M_{2}=\left\{\beta_{2}^{\prime}, \ldots, \beta_{N-1}^{\prime}\right\} \cup\left\{\beta_{1}^{\prime}+p_{i}, \beta_{N}^{\prime}-p_{i}\right\}$. Moreover we can show that

$$
\begin{aligned}
& \left\{\beta_{1}, \ldots, \beta_{p-1}\right\}=\left\{\beta_{2}^{\prime}, \ldots, \beta_{p-1}^{\prime}\right\} \cup\left\{\beta_{N}^{\prime}-p_{i}\right\}, \\
& \left\{\beta_{p}, \ldots, \beta_{N}\right\}=\left\{\beta_{p}^{\prime}, \ldots, \beta_{N-1}^{\prime}\right\} \cup\left\{\beta_{1}^{\prime}+p_{i}\right\} .
\end{aligned}
$$

Since $\beta-\alpha<p_{i}$ one has $\beta_{N}^{\prime}-p_{i}=\beta-p_{i}<\alpha=\alpha_{p}^{\prime} \leq \beta_{p}^{\prime}$. This implies (30). Since $\alpha_{N}^{\prime}-\alpha_{1}^{\prime} \leq p_{i}$ one has $\beta_{1}^{\prime}+p_{i} \geq \beta_{1}^{\prime}+\alpha_{N}^{\prime}-\alpha_{1}^{\prime}=\alpha_{N}^{\prime} \geq \beta_{N-1}^{\prime}$. This implies (31), or more precisely $\beta_{k}=\beta_{k}^{\prime}(p \leq k \leq N-1)$ and $\beta_{N}=\beta_{1}^{\prime}+p_{i}$. Clearly the condition $\alpha_{p} \leq \beta_{p} \leq \ldots \leq \alpha_{N} \leq \beta_{N}$ holds. Noting that $\alpha_{k}^{\prime}=\beta_{k}^{\prime}$ for $1 \leq k \leq p-1$, it is also easy to see $\alpha_{1} \leq \beta_{1} \leq \ldots \leq \alpha_{p-1} \leq \beta_{p-1}$. Finally we find that $\beta_{p-1}=\max \left(\beta_{p-1}^{\prime}, \beta_{N}^{\prime}-p_{i}\right) \leq \alpha_{p}^{\prime}=\alpha_{p}$ and $\beta_{N}-\alpha_{1}=\beta_{1}^{\prime}+p_{i}-\alpha_{1}^{\prime}=p_{i}$, completing the proof of item (ii).

Proof of item (iii): Denote by $M_{1}^{\prime}=\left\{\alpha_{1}^{\prime}, \ldots, \alpha_{N}^{\prime}\right\}$ and $M_{2}^{\prime}=\left\{\beta_{1}^{\prime}, \ldots, \beta_{N}^{\prime}\right\}$ the $M_{1}, M_{2}$ that one has just after step IS2 in the $i$-th turn. We assume that the conditions $\alpha_{1}^{\prime} \leq \beta_{1}^{\prime} \leq \ldots \leq \alpha_{N}^{\prime} \leq \beta_{N}^{\prime}$ and $\beta_{N}^{\prime}-\alpha_{1}^{\prime} \leq p_{i}$ are satisfied. Let $M_{1}=\left\{\alpha_{1}, \ldots, \alpha_{N}\right\}$ and $M_{2}=\left\{\beta_{1}, \ldots, \beta_{N}\right\}$ be the $M_{1}, M_{2}$ that one has just after step IS3 in the $i$-th turn. Clearly the condition $\alpha_{1}<\beta_{1}<\cdots<\alpha_{N}<\beta_{N}$ holds. It is also easy to see that $\alpha_{1}=\alpha_{1}^{\prime}$ and $\beta_{N}=\beta_{N}^{\prime}+\left(\ell_{i}-\ell_{i+1}\right)\left(2 \sum_{k=1}^{i} m_{k}-1\right)=\beta_{N}^{\prime}+p_{i+1}-p_{i}+\ell_{i+1}-\ell_{i}$. Hence $\beta_{N}-\alpha_{1} \leq p_{i+1}+\ell_{i+1}-\ell_{i}<p_{i+1}$.

Proof of item (iv): Let $M_{1}=\left\{\alpha_{1}, \ldots, \alpha_{N}\right\}$ be the $M_{1}$ that one has just before step IS4. The $\alpha_{1}$ is given as $\alpha_{1}=x_{i, 1}$ for some $i$. Thus we have $\alpha_{1} \geq 0$ by (26) . The $\alpha_{N}$ is given by the following formula for some $i$ :

$$
\begin{aligned}
\alpha_{N} & =x_{i, m_{i}}+2 \sum_{k=i}^{s}\left(\ell_{k}-\ell_{k+1}\right)\left(\sum_{h=1}^{k} m_{h}-1\right) \\
& =x_{i, m_{i}}+\sum_{k=i}^{s}\left(\left(p_{k+1}-p_{k}\right)-2\left(\ell_{k}-\ell_{k+1}\right)\right) \\
& =x_{i, m_{i}}+L-p_{i}-2 \ell_{i} .
\end{aligned}
$$

Thus we have $\alpha_{N} \leq L-1$ by (27).

\subsection{Statistics preserving bijection and the partition function}

We classify the elements of $\Phi\left(P_{L, M}\right)$ according to their soliton contents. As a result of Lemma 29 to be presented in the next section we have

$$
\left|\Omega^{\circ}(\lambda, L)\right|=\frac{L}{m_{1}}\left(\begin{array}{c}
p_{1}+m_{1}-1 \\
m_{1}-1
\end{array}\right) \prod_{i=2}^{s}\left(\begin{array}{c}
p_{i}+m_{i}-1 \\
m_{i}
\end{array}\right) .
$$

By using this expression one can show that $\sum_{\lambda \vdash M}\left|\Omega^{\circ}(\lambda, L)\right|=\left(\begin{array}{c}L \\ M\end{array}\right)$, while Lemma 23 implies that $\bigsqcup_{\lambda \vdash M}\{\lambda\} \times \Omega^{\circ}(\lambda, L) \subset \Phi\left(P_{L, M}\right)$, which leads to

$$
\sum_{\lambda \vdash M}\left|\Omega^{\circ}(\lambda, L)\right|=\left|\bigsqcup_{\lambda \vdash M}\{\lambda\} \times \Omega^{\circ}(\lambda, L)\right| \leq\left|\Phi\left(P_{L, M}\right)\right|=\left|P_{L, M}\right|=\left(\begin{array}{c}
L \\
M
\end{array}\right) .
$$


Hence $\bigsqcup_{\lambda \vdash M}\{\lambda\} \times \Omega^{\circ}(\lambda, L)=\Phi\left(P_{L, M}\right)$. In other words we have:

Theorem 24 The map $\Phi$ gives a bijection between $P_{L, M}$ and $\bigsqcup_{\lambda \vdash M}\{\lambda\} \times \Omega^{\circ}(\lambda, L)$.

This bijection is statistics preserving in the following sense.

Given a path $p \in P_{L, M}$ let $M_{1}=\left\{\alpha_{1}, \ldots, \alpha_{N}\right\}$ be its associated multiset, i.e. for $p=b_{1} \cdots b_{L}$ we let $\alpha \in\{0, \ldots, L-1\}$ belong to $M_{1}$ if and only if the condition $\left(b_{\alpha}, b_{\alpha+1}\right)=(1,2)$ is satisfied, where we interpret $b_{0}=1$. Noting that $E_{\text {path }}(p)=\sum_{i=1}^{N} \alpha_{i}$ it is easy to see that the algorithm of the inverse scattering transform implies the following result.

Theorem 25 Given $p \in P_{L, M}$ let $\Phi(p)=(\lambda, \boldsymbol{x})$. Then $E_{\mathrm{path}}(p)=E_{\mathrm{RC}}(\lambda, \boldsymbol{x})$.

Proof: The energy $E_{\text {path }}(p)$ is equal to sum of the elements of the multiset $M_{1}$. Consider how $M_{1}$ has been constructed in the algorithm of the inverse scattering transform. One finds that step IS1 is responsible for the term $\sum_{i=1}^{s} \sum_{j=1}^{m_{i}} x_{i, j}$ in the definition of $E_{\mathrm{RC}}(\lambda, \boldsymbol{x})$, and step IS3 is for the $\psi(\lambda)$. (See the way of its calculation presented above Example 1.) The other steps are irrelevant.

Let $P_{L, \lambda}^{\circ}=\Phi^{-1}\left(\{\lambda\} \times \Omega^{\circ}(\lambda, L)\right)$, which is the set of all paths of length $L$ and with soliton content $\lambda$. Then $\Phi$ gives a statistics preserving bijection between $P_{L, \lambda}^{\circ}$ and $\{\lambda\} \times \Omega^{\circ}(\lambda, L)$. We consider the partition function for the periodic soliton cellular automaton over $P_{L, \lambda}^{\circ}$. By Theorem 25 we have

$$
\sum_{p \in P_{L, \lambda}^{\circ}} q^{E_{\mathrm{path}}(p)}=\sum_{\boldsymbol{x} \in \Omega^{\circ}(\lambda, L)} q^{E_{\mathrm{RC}}(\lambda, \boldsymbol{x})}=q^{\psi(\lambda)} \sum_{\boldsymbol{x} \in \Omega^{\circ}(\lambda, L)} q^{|\boldsymbol{x}|},
$$

where $|\boldsymbol{x}|=\sum_{i=1}^{s} \sum_{k=1}^{m_{i}} x_{i, k}$. In the next section we show that the sum $\sum_{\boldsymbol{x} \in \Omega^{\circ}(\lambda, L)} q^{|\boldsymbol{x}|}$ admits a fermionic expression.

\subsection{The case of non-periodic paths}

In this subsection we briefly review direct and inverse scattering transforms for nonperiodic paths [9]. We denote by $\Psi: p \mapsto(\lambda, \boldsymbol{x})$ the direct scattering transform, where $p$ is a path of length $L$. Let $M_{1}, M_{2}, j, k, D, S$ be those introduced just above the algorithm DS0-DS4 for the map $\Phi$ in subsection 4.2. The algorithm for the map $\Psi$ is given as follows.

ds1 While $M_{1} \cap M_{2}=\emptyset$, continue replacing $\left(j, k, M_{1}, M_{2}\right)$ by $(j+1, k-$ $\left.2\left|M_{1}\right|, \mathcal{O}_{0}^{-1}\left(M_{1}\right), \mathcal{O}_{1}^{-1}\left(M_{2}\right)\right)$.

ds2 If $M_{1} \cap M_{2} \neq \emptyset$, then set $S=M_{1} \cap M_{2}$ and replace $\left(M_{1}, M_{2}\right)$ by $\left(M_{1} \backslash S, M_{2} \backslash S\right)$.

ds3 Pre-pend $\{S, j\}$ to $D$ and set $S=\emptyset$. If $M_{1}=\emptyset$ then stop. Otherwise go to step ds1.

At the end we obtain such type of data $D=\left\{\left\{S_{1}, j_{1}\right\},\left\{S_{2}, j_{2}\right\}, \ldots,\left\{S_{s}, j_{s}\right\}\right\}$ for some $s$, where $S_{1}, \ldots, S_{s}$ are multisets, $j_{1}, \ldots, j_{s}$ are positive integers. Let $\ell_{i}=j_{i}, m_{i}=\left|S_{i}\right|$ and $\boldsymbol{x}_{i}=S_{i}$. By Figure 1 the data $\left\{\left(\ell_{i}, m_{i}\right)\right\}_{1 \leq i \leq s}$ determines a Young diagram $\lambda$. 
Fermionic partition functions for a periodic soliton cellular automaton

Let $M_{1}, M_{2}, S_{i}$ be those introduced just above the algorithm IS1-IS4 for the map $\Phi^{-1}$ in subsection 4.3. The inverse map $\Psi^{-1}$ is given as follows. To begin with we set $M_{1}=M_{2}=\emptyset$ and $i=1$. Then run the following algorithm where we interpret $\ell_{s+1}=0$.

is1 Replace $M_{1}$ by $M_{1} \cup S_{i}$ and $M_{2}$ by $M_{2} \cup S_{i}$.

is2 Replace $M_{1}$ by $\mathcal{O}_{0}^{\ell_{i}-\ell_{i+1}}\left(M_{1}\right)$ and $M_{2}$ by $\mathcal{O}_{1}^{\ell_{i}-\ell_{i+1}}\left(M_{2}\right)$. If $i<s$, then replace $i$ by $i+1$ and go back to step is1. If $i=s$, then stop.

By using the method explained just below (25) we obtain a path $p$ from the final forms of the $M_{1}, M_{2}$.

Theorem 26 ([9]) The map $\Psi$ gives a bijection between $P_{L, M}$ (resp. $P_{L, M}^{+}$) and $\bigsqcup_{\lambda \vdash M}\{\lambda\} \times \Omega(\lambda, L)$ (resp. $\bigsqcup_{\lambda \vdash M}\{\lambda\} \times \Omega^{+}(\lambda, L)$ ).

Theorem $27([\mathbf{9}])$ Given $p \in P_{L, M}$ let $\Psi(p)=(\lambda, \boldsymbol{x})$. Then $E_{\mathrm{path}}(p)=E_{\mathrm{RC}}(\lambda, \boldsymbol{x})$.

\section{Configuration sums over modified rigged configurations}

The l's in this section should be interpreted as $2 \ell$ 's in the previous section.

Lemma 28 For any positive integers $l, m, n, p$ the following relation holds

$$
\sum_{x_{1}, \ldots, x_{m}, y} q^{x_{1}+\cdots+x_{m}+y}=[p+l(n+m)]\left[\begin{array}{c}
p+m-1 \\
m
\end{array}\right],
$$

where the sum is taken over all integers $x_{1}, \ldots, x_{m}, y$ satisfying

$$
\begin{aligned}
& 0 \leq x_{1} \leq \ldots \leq x_{m} \leq p+\min \left(x_{1}, l-1\right), \\
& n \max \left(0, x_{m}-p\right) \leq y \leq p-1+n \min \left(l, x_{1}\right) .
\end{aligned}
$$

Proof. Set $\lambda_{1}=x_{m}-x_{1}, \lambda_{2}=x_{m-1}-x_{1}, \ldots, \lambda_{m-1}=x_{2}-x_{1}$ and $\lambda=\left(\lambda_{1}, \ldots, \lambda_{m-1}\right)$. Then

$$
\sum_{x_{1}, \ldots, x_{m}, y} q^{x_{1}+\cdots+x_{m}+y}=\sum_{x_{1}, y, \lambda} q^{m x_{1}+y+|\lambda|},
$$

where the sum in RHS is over all integers $x_{1}, y$ satisfying

$$
\begin{aligned}
& 0 \leq x_{1} \leq p+l-\lambda_{1}-1, \\
& n \max \left(0, x_{1}+\lambda_{1}-p\right) \leq y \leq p-1+n \min \left(l, x_{1}\right),
\end{aligned}
$$

and over all partitions $\lambda$ with at most $m-1$ parts, the largest part $\leq p$. For any fixed $x_{1}, x_{m}$ the sum $\sum_{y:(34} q^{y}$ amounts to

$$
\begin{array}{cl}
{\left[p+n x_{1}\right],} & \left(0 \leq x_{1} \leq \min \left(l, p-\lambda_{1}\right)-1\right) \\
{[p+n l],} & \left(l \leq x_{1} \leq p-\lambda_{1}-1\right) \\
q^{n x_{1}-n\left(p-\lambda_{1}\right)}\left[p+n\left(p-\lambda_{1}\right)\right], & \left(p-\lambda_{1} \leq x_{1} \leq l-1\right) \\
q^{n x_{1}-n\left(p-\lambda_{1}\right)}\left[p+n\left(p-\lambda_{1}+l-x_{1}\right)\right] . & \left(\max \left(l, p-\lambda_{1}\right) \leq x_{1} \leq p+l-\lambda_{1}-1\right)
\end{array}
$$


Fermionic partition functions for a periodic soliton cellular automaton

So if $l<p-\lambda_{1}$ the the sum $\sum_{x_{1}, y} q^{m x_{1}+y}$ amounts to the sum of

$$
\begin{gathered}
\sum_{0 \leq x_{1} \leq l-1} q^{m x_{1}}\left[p+n x_{1}\right]=\frac{1}{1-q}\left(\frac{[m l]}{[m]}-q^{p} \frac{[(m+n) l]}{[m+n]}\right), \\
\sum_{l \leq x_{1} \leq p-\lambda_{1}-1} q^{m x_{1}}[p+n l]=\frac{1}{1-q}\left(\left(1-q^{p+l n}\right) \frac{\left[m\left(p-\lambda_{1}\right)\right]-[m l]}{[m]}\right), \\
\sum_{p-\lambda_{1} \leq x_{1} \leq p+l-\lambda_{1}-1} q^{(m+n) x_{1}-n\left(p-\lambda_{1}\right)}\left[p+n\left(p-\lambda_{1}+l-x_{1}\right)\right] \\
=\frac{1}{1-q}\left(q^{m\left(p-\lambda_{1}\right)} \frac{[(m+n) l]}{[m+n]}-q^{p+l n+m\left(p-\lambda_{1}\right)} \frac{[m l]}{[m]}\right)
\end{gathered}
$$

and if $l>p-\lambda_{1}$ it leads to sum of

$$
\begin{gathered}
\sum_{0 \leq x_{1} \leq p-\lambda_{1}-1} q^{m x_{1}}\left[p+n x_{1}\right]=\frac{1}{1-q}\left(\frac{\left[m\left(p-\lambda_{1}\right)\right]}{[m]}-q^{p} \frac{\left[(m+n)\left(p-\lambda_{1}\right)\right]}{[m+n]}\right), \\
\sum_{p-\lambda_{1} \leq x_{1} \leq l-1} q^{(m+n) x_{1}-n\left(p-\lambda_{1}\right)}\left[p+n\left(p-\lambda_{1}\right)\right] \\
=\frac{1}{1-q}\left(\left(q^{-n\left(p-\lambda_{1}\right)}-q^{p}\right) \frac{[(m+n) l]-\left[(m+n)\left(p-\lambda_{1}\right)\right]}{[m+n]}\right), \\
\sum_{l \leq x_{1} \leq p+l-\lambda_{1}-1} q^{(m+n) x_{1}-n\left(p-\lambda_{1}\right)}\left[p+n\left(p-\lambda_{1}+l-x_{1}\right)\right] \\
=\frac{1}{1-q}\left(q^{-n\left(p-\lambda_{1}\right)+(m+n) l} \frac{\left[(m+n)\left(p-\lambda_{1}\right)\right]}{[m+n]}-q^{p+(m+n) l} \frac{\left[m\left(p-\lambda_{1}\right)\right]}{[m]}\right) .
\end{gathered}
$$

It is easy to see that in both cases one has

$$
\sum_{x_{1}, y} q^{m x_{1}+y}=\frac{1}{1-q}\left(\frac{[l(m+n)]}{[m+n]}\left(q^{m\left(p-\lambda_{1}\right)}-q^{p}\right)+\frac{[p+l(m+n)]}{[m]}\left(1-q^{m\left(p-\lambda_{1}\right)}\right)\right) .
$$

From this expression and by the following formulas we obtain the desired relation.

$$
\sum_{\lambda} q^{|\lambda|}=\left[\begin{array}{c}
p+m-1 \\
m-1
\end{array}\right], \quad \sum_{\lambda} q^{|\lambda|+m\left(p-\lambda_{1}\right)}=q^{p}\left[\begin{array}{c}
p+m-1 \\
m-1
\end{array}\right] .
$$

Let $l_{1}, \ldots, l_{s}, m_{1}, \ldots, m_{s}$ be positive integers. We impose $l_{1}>\cdots>l_{s}$. Given any integer $p_{1} \geq 0$ we define $p_{i}(2 \leq i \leq s)$ by $p_{i}=p_{i-1}+\left(m_{1}+\cdots+m_{i-1}\right)\left(l_{i-1}-l_{i}\right)$. Let $p_{s+1}=p_{s}+\left(m_{1}+\cdots+m_{s}\right) l_{s}$

Let $\boldsymbol{x}=\left(x_{1,1}, \ldots, x_{1, m_{1}}, x_{2,1}, \ldots, x_{2, m_{2}}, \ldots, x_{s, 1}, \ldots, x_{s, m_{s}}\right) \in \mathbb{Z}^{\mathcal{N}}$ where $\mathcal{N}=$ $m_{1}+\cdots+m_{s}$. Then let

$$
\Omega_{s}=\left\{\begin{array}{l|l}
\boldsymbol{x} \in \mathbb{Z}^{\mathcal{N}} & \begin{array}{l}
0 \leq x_{i, 1} \leq \cdots \leq x_{i, m_{i}} \leq p_{i}+\min \left(x_{i, 1}, l_{i}-1\right) \\
\text { for } 1 \leq i \leq s, \\
x_{j, m_{j}}-p_{j} \leq x_{i, 1} \text { for } 1 \leq i<j \leq s, \\
x_{i, m_{i}} \leq p_{i}+l_{i}-l_{j}+x_{j, 1}-1 \text { for } 1 \leq i<j \leq s
\end{array}
\end{array}\right\} .
$$

If we set $l_{i}=2 \ell_{i}$ and $p_{s+1}=L$, then $\Omega_{s}$ coincides with $\Omega^{\circ}(\lambda, L)$ in the previous section. 
Fermionic partition functions for a periodic soliton cellular automaton

Lemma 29 The following relation holds:

$$
\sum_{\boldsymbol{x} \in \Omega_{s}} q^{|\boldsymbol{x}|}=\frac{\left[p_{s+1}\right]}{\left[m_{1}\right]}\left[\begin{array}{c}
p_{1}+m_{1}-1 \\
m_{1}-1
\end{array}\right] \prod_{i=2}^{s}\left[\begin{array}{c}
p_{i}+m_{i}-1 \\
m_{i}
\end{array}\right],
$$

where $|\boldsymbol{x}|=\sum_{i=1}^{s} \sum_{k=1}^{m_{i}} x_{i, k}$.

Proof. It is done by induction on $s$. When $s=1$ the relation becomes

$$
\sum_{\boldsymbol{x} \in \Omega_{1}} q^{x_{1}+\cdots+x_{m}}=\frac{[p+m l]}{[m]}\left[\begin{array}{c}
p+m-1 \\
m-1
\end{array}\right]
$$

where

$$
\Omega_{1}=\left\{\boldsymbol{x} \in \mathbb{Z}^{m} \mid 0 \leq x_{1} \leq \cdots \leq x_{m} \leq p+\min \left(x_{1}, l-1\right)\right\} .
$$

It is proved by using

$$
\sum_{\boldsymbol{x} \in \Omega_{1}, x_{1}<l-1} q^{x_{1}+\cdots+x_{m}}=\left(1+q^{m}+\cdots+q^{(l-2) m}\right)\left[\begin{array}{c}
p+m-1 \\
m-1
\end{array}\right]
$$

and

$$
\sum_{\boldsymbol{x} \in \Omega_{1}, x_{1} \geq l-1} q^{x_{1}+\cdots+x_{m}}=q^{(l-1) m}\left[\begin{array}{c}
p+m \\
m
\end{array}\right] .
$$

For $s>1$ we assume the claim of Lemma but $s$ has been replaced by $s-1$. More precisely, we set

$$
\boldsymbol{x}^{\prime}=\left(x_{1,1}, \ldots, x_{1, m_{1}}, x_{2,1}, \ldots, x_{2, m_{2}}, \ldots, x_{s-1,1}, \ldots, x_{s-1, m_{s-1}}\right) \in \mathbb{Z}^{n}
$$

where $n=m_{1}+\cdots+m_{s-1}$ and let

$$
\Omega_{s-1}=\left\{\begin{array}{l|l}
\boldsymbol{x}^{\prime} \in \mathbb{Z}^{n} & \begin{array}{l}
0 \leq x_{i, 1} \leq \cdots \leq x_{i, m_{i}} \leq p_{i}+\min \left(x_{i, 1}, l_{i}-1\right) \\
\text { for } 1 \leq i \leq s-1, \\
x_{j, m_{j}}-p_{j} \leq x_{i, 1} \text { for } 1 \leq i<j \leq s-1, \\
x_{i, m_{i}} \leq p_{i}+l_{i}-l_{j}+x_{j, 1}-1 \text { for } 1 \leq i<j \leq s-1
\end{array}
\end{array}\right\} .
$$

Then the following relation is supposed to hold:

$$
\sum_{\boldsymbol{x}^{\prime} \in \Omega_{s-1}} q^{\left|\boldsymbol{x}^{\prime}\right|}=\frac{\left[p_{s-1}+n l_{s-1}\right]}{\left[m_{1}\right]}\left[\begin{array}{c}
p_{1}+m_{1}-1 \\
m_{1}-1
\end{array}\right] \prod_{i=2}^{s-1}\left[\begin{array}{c}
p_{i}+m_{i}-1 \\
m_{i}
\end{array}\right] \text {. }
$$

Note that $p_{s-1}+n l_{s-1}=p_{s}+n l_{s}$.

We denote by $\pi$ the projection from $\mathbb{Z}^{n} \times \mathbb{Z}^{m_{s}}$ onto $\mathbb{Z}^{n}$. Note that $\pi\left(\Omega_{s}\right) \subset \mathbb{Z}^{n}$ does not coincide with $\Omega_{s-1} \subset \mathbb{Z}^{n}$. To see their differences, set $x_{i}=x_{s, i}\left(1 \leq i \leq m_{s}\right), m=$ $m_{s}, l=l_{s}, p=p_{s}$, and $\lambda_{1}=x_{m}-x_{1}$. Let $\left.\Omega_{s}\right|_{x_{1}, \lambda_{1}}$ be the set of all $\boldsymbol{x} \in \Omega_{s}$ with fixed $x_{1}, \lambda_{1}$. Then for any $\boldsymbol{x}^{\prime} \in \pi\left(\left.\Omega_{s}\right|_{x_{1}, \lambda_{1}}\right) \subset \mathbb{Z}^{n}$ the conditions

$$
\begin{aligned}
& x_{1}+\lambda_{1}-p \leq x_{i, 1}, \\
& x_{i, m_{i}} \leq p_{i}+l_{i}-l+x_{1}-1,
\end{aligned}
$$


for $1 \leq i \leq s-1$ are imposed, in addition to

$$
\begin{aligned}
& 0 \leq x_{i, 1} \leq \cdots \leq x_{i, m_{i}} \leq p_{i}+\min \left(x_{i, 1}, l_{i}-1\right) \quad \text { for } \quad 1 \leq i \leq s-1 \\
& x_{j, m_{j}}-p_{j} \leq x_{i, 1} \\
& x_{i, m_{i}} \leq p_{i}+l_{i}-l_{j}+x_{j, 1}-1
\end{aligned} \quad \text { for } \quad 1 \leq i<j \leq s-1 .
$$

Here we wrote the entries of $\boldsymbol{x}^{\prime}$ as in (40). Note that the latter conditions (44), (45) are those for $\Omega_{s-1}$. We claim that if the sum over $\boldsymbol{x}^{\prime} \in \Omega_{s-1}$ in (41) is replaced by the sum over $\boldsymbol{x}^{\prime} \in \pi\left(\left.\Omega_{s}\right|_{x_{1}, \lambda_{1}}\right)$, then the only change thereby caused is within the factor $\left[p_{s-1}+n l_{s-1}\right]$ in RHS. More precisely, this factor is replaced by one of (35)-(38) depending on the conditions on $x_{1}$ and $\lambda_{1}$ :

$$
\sum_{\boldsymbol{x}^{\prime} \in \pi\left(\left.\Omega_{s}\right|_{x_{1}, \lambda_{1}}\right)} q^{\left|\boldsymbol{x}^{\prime}\right|}=Q \cdot \begin{cases}{\left[p+n x_{1}\right]} & \left(0 \leq x_{1} \leq \min \left(l, p-\lambda_{1}\right)-1\right) \\ {[p+n l]} & \left(l \leq x_{1} \leq p-\lambda_{1}-1\right) \\ q^{n\left(x_{1}+\lambda_{1}-p\right)}\left[p+n\left(p-\lambda_{1}\right)\right] & \left(p-\lambda_{1} \leq x_{1} \leq l-1\right) \\ q^{n\left(x_{1}+\lambda_{1}-p\right)}\left[p+n\left(p-\lambda_{1}+l-x_{1}\right)\right] & \left(\max \left(l, p-\lambda_{1}\right) \leq x_{1} \leq p+l-\lambda_{1}-1\right)\end{cases}
$$

where

$$
Q=\frac{1}{\left[m_{1}\right]}\left[\begin{array}{c}
p_{1}+m_{1}-1 \\
m_{1}-1
\end{array}\right] \prod_{i=2}^{s-1}\left[\begin{array}{c}
p_{i}+m_{i}-1 \\
m_{i}
\end{array}\right] .
$$

Admitting this claim, the Lemma immediately follows as a result of Lemma 28 .

$$
\begin{aligned}
& \sum_{\boldsymbol{x} \in \Omega_{s}} q^{|\boldsymbol{x}|}=\sum_{x_{1}, \ldots, x_{m}:(33)}\left(q^{x_{1}+\cdots+x_{m}} \sum_{\boldsymbol{x}^{\prime} \in \pi\left(\left.\Omega_{s}\right|_{x_{1}, \lambda_{1}}\right)} q^{\left|\boldsymbol{x}^{\prime}\right|}\right) \\
& \sum_{\boldsymbol{x}^{\prime} \in \pi\left(\left.\Omega_{s}\right|_{x_{1}, \lambda_{1}}\right)} q^{\left|\boldsymbol{x}^{\prime}\right|}=Q \cdot \sum_{y:(34} q^{y}, \\
& \sum_{x_{1}, \ldots, x_{m}: 33, y: 34} q^{x_{1}+\cdots+x_{m}+y}=\left[p_{s}+l_{s}\left(m_{1}+\cdots+m_{s}\right)\right]\left[\begin{array}{c}
p_{s}+m_{s}-1 \\
m_{s}
\end{array}\right] \text {. }
\end{aligned}
$$

We suppose $0 \leq x_{1} \leq \min \left(l, p-\lambda_{1}\right)-1$ and show how the factor $\left[p_{s-1}+n l_{s-1}\right]$ is replaced by $\left[p+n x_{1}\right]$. The condition (42) is now satisfied as $x_{1}+\lambda_{1}-p \leq-1<0 \leq x_{i, 1}$ by (44). Based on the following arguments, it is easy to see that $\pi\left(\left.\Omega_{s}\right|_{x_{1}, \lambda_{1}}\right)$ is obtained from $\Omega_{s-1}$ by replacing $l_{i}$ with $l_{i}-l+x_{1}$ for all $1 \leq i \leq s-1$. First of all, any simultaneous constant shift of $l_{i}$ 's does not change $p_{i}$ 's. Hence it leaves the condition (45) unchanged, as well as all the factors in (41) except the $\left[p_{s-1}+n l_{s-1}\right]$. The condition (43) is achieved by the above replacement $l_{i} \rightarrow l_{i}-l+x_{1}$ in (44). Therefore the only change of (41) caused by the replacement of $\Omega_{s-1}$ with $\pi\left(\left.\Omega_{s}\right|_{x_{1}, \lambda_{1}}\right)$ is given by replacing $\left[p_{s-1}+n l_{s-1}\right]$ with $\left[p_{s-1}+n\left(l_{s-1}-l+x_{1}\right)\right]=\left[p+n x_{1}\right]$.

Suppose $l \leq x_{1} \leq p-\lambda_{1}-1$. The condition (42) is satisfied as above, and (43) is now satisfied as $x_{i, m_{i}} \leq p_{i}+l_{i}-1 \leq p_{i}+l_{i}-1+x_{1}-l$ by (44). Hence no changes are caused by the replacement of $\Omega_{s-1}$ with $\pi\left(\left.\Omega_{s}\right|_{x_{1}, \lambda_{1}}\right)$. Note that $\left[p_{s-1}+n l_{s-1}\right]=[p+n l]$.

Suppose $p-\lambda_{1} \leq x_{1} \leq l-1$. By the conditions (42)-(44) we have

$$
x_{1}+\lambda_{1}-p \leq x_{i, 1} \leq \cdots \leq x_{i, m_{i}} \leq p_{i}+\min \left(x_{i, 1}, l_{i}-l+x_{1}-1\right),
$$


for $1 \leq i \leq s-1$. By replacing $x_{i, j}$ with $x_{i, j}+x_{1}+\lambda_{1}-p$ this condition becomes

$$
0 \leq x_{i, 1} \leq \cdots \leq x_{i, m_{i}} \leq p_{i}+\min \left(x_{i, 1}, \tilde{l}_{i}-1\right)
$$

where $\tilde{l}_{i}=l_{i}-l-\lambda_{1}+p$. Note that the simultaneous constant shift $x_{i, j} \rightarrow x_{i, j}+x_{1}+\lambda_{1}-p$ for $\left\{x_{i, j}\right\}_{1 \leq i \leq s-1,1 \leq j \leq m_{i}}$ makes the change $q^{\left|\boldsymbol{x}^{\prime}\right|} \rightarrow q^{\left|\boldsymbol{x}^{\prime}\right|+n\left(x_{1}+\lambda_{1}-p\right)}$ and leaves the condition (45) unchanged. Hence

$$
\sum_{\boldsymbol{x}^{\prime} \in \pi\left(\left.\Omega_{s}\right|_{x_{1}, \lambda_{1}}\right)} q^{\left|\boldsymbol{x}^{\prime}\right|}=q^{n\left(x_{1}+\lambda_{1}-p\right)} \sum_{\boldsymbol{x}^{\prime}: 45, \text {, 46 }} q^{\left|\boldsymbol{x}^{\prime}\right|} .
$$

By the replacement $l_{i} \rightarrow \tilde{l}_{i}$ the condition (44) becomes (46), and the condition (45) is left unchanged. Hence

$$
\sum_{\boldsymbol{x}^{\prime}: 457,46} q^{\left|\boldsymbol{x}^{\prime}\right|}=Q \cdot\left[p_{s-1}+n \tilde{l}_{s-1}\right]=Q \cdot\left[p+n\left(p-\lambda_{1}\right)\right] .
$$

Suppose $\max \left(l, p-\lambda_{1}\right) \leq x_{1} \leq p+l-\lambda_{1}-1$. The condition (43) is now satisfied as $x_{i, m_{i}} \leq p_{i}+l_{i}-1 \leq p_{i}+l_{i}-1+x_{1}-l$ by (44). By the conditions (42), (44) we have

$$
x_{1}+\lambda_{1}-p \leq x_{i, 1} \leq \cdots \leq x_{i, m_{i}} \leq p_{i}+\min \left(x_{i, 1}, l_{i}-1\right),
$$

for $1 \leq i \leq s-1$. By replacing $x_{i, j}$ with $x_{i, j}+x_{1}+\lambda_{1}-p$ this condition becomes

$$
0 \leq x_{i, 1} \leq \cdots \leq x_{i, m_{i}} \leq p_{i}+\min \left(x_{i, 1}, \hat{l}_{i}-1\right)
$$

where $\hat{l}_{i}=l_{i}-x_{1}-\lambda_{1}+p$. By the same argument in the previous paragraph, we have

$$
\begin{aligned}
& \sum_{\boldsymbol{x}^{\prime} \in \pi\left(\left.\Omega_{s}\right|_{x_{1}, \lambda_{1}}\right)} q^{\left|\boldsymbol{x}^{\prime}\right|}=q^{n\left(x_{1}+\lambda_{1}-p\right)} \sum_{\boldsymbol{x}^{\prime}: 45} q^{\left|\boldsymbol{x}^{\prime}\right|}, \\
& \sum_{\boldsymbol{x}^{\prime}: \text {,47 }} q^{\left|\boldsymbol{x}^{\prime}\right|}=Q \cdot\left[p_{s-1}+n \hat{l}_{s-1}\right]=Q \cdot\left[p+n\left(p-\lambda_{1}+l-x_{1}\right)\right] .
\end{aligned}
$$

Acknowledgement. This work is supported by Grants-in-Aid for Scientific Research No. 21540209 and No. 25540241 from JSPS.

[1] Kashiwara M and Miwa T (eds) 2000 Physical Combinatorics, Prog. in Math. 191 (Boston: Birkhäuser)

[2] Kuniba A and Okado M (eds) 2007 Combinatorial aspect of integrable systems, Math. Soc. Jpn. Memoirs 17 (Tokyo: MSJ)

[3] Baxter R J 2007 Exactly solved models in statistical mechanics (New York: Dover)

[4] Kerov S V, Kirillov A N and Reshetikhin N Yu 1988 Combinatorics, the Bethe ansatz and representations of the symmetric group. J. Soviet Math. 41 916-924

[5] Kirillov A N and Reshetikhin N Yu 1988 The Bethe ansatz and the combinatorics of Young tableaux, J. Soviet Math. 41 925-955

[6] Bethe H A 1931 Zur Theorie der Metalle. I. Eigenwerte und Eigenfunktionen der linearen Atomkettee, Z. Phys. 71 205-226

[7] Takahashi D, Satsuma J 1990 A soliton cellular automaton, J. Phys. Soc. Japan 59 3514-3519

[8] Kuniba A, Okado M, Takagi T and Yamada Y 2003 Vertex operators and canonical partition function of box-ball system (in Japanese), RIMS Kôkyûroku, 1302 91-107

[9] Takagi T 2005 Inverse scattering method for a soliton cellular automaton Nucl. Phys. B 707 $577-601$ 
[10] Yoshihara D, Yura F and Tokihiro T 2003 Fundamental cycle of a periodic box-ball system, J. Phys. A: Math. Gen. 36 99-121

[11] Kuniba A Takagi T and Takenouchi A 2006 Bethe ansatz and inverse scattering transform in a periodic box-ball system Nucl. Phys. B 747 [PM] 354-397

[12] Kuniba A and Nakanishi T 2000 The Bethe equation at $q=0$, the Möbius inversion formula, and weight multiplicities: I. The $s l(2)$ case, Prog. in Math. 191 185-216

[13] Andrews G E 1984 The Theory of Partitions (Cambridge: Cambridge University Press.)

[14] Stanley R P 1999 Enumerative combinatorics vol.1 (Cambridge: Cambridge University Press.)

[15] Kirillov A N and Sakamoto R 2009 Relationships between two approaches: rigged configurations and 10-eliminations, Lett. in Math. Phys 89 51-65

[16] Mada J Idzumi M and Tokihiro T 2006 On the initial value problem of a periodic box-ball system J. Phys. A: Math. Gen. 39 L617 